\title{
Unsteady three-dimensional sources for a two-layer fluid of finite depth and their applications
}

\author{
Thai C. Nguyen • Ronald W. Yeung
}

Received: 30 April 2010 / Accepted: 27 June 2010 / Published online: 28 December 2010

(C) The Author(s) 2010. This article is published with open access at Springerlink.com

\begin{abstract}
To our friend Ernie Tuck, in celebration of his multi-faceted talents. The velocity potentials of various unsteady point sources are derived in this paper for a two-layer fluid of finite depth. Two-layer fluids are often used to study effects of density stratification on hydrodynamics of marine systems. The sources here are restricted to the upper fluid layer and the potentials of the induced flows are given for the whole fluid domain. The velocity potentials of a transient source of arbitrary strength and in arbitrary three-dimensional motion are derived first. The potentials of a time-harmonic source without forward speed, and then with forward speed, are obtained from the transient source by specifying the appropriate source strength and motion. These potentials are fundamental to the analyses of various types of body motion in finite water depths under the influence of surface and interfacial waves. As a sample application, a numerical solution of the radiation and diffraction problem for a floating rectangular barge is presented. The results indicate that internal waves can have a strong effect on the motions of the floating barge over a wide range of incident-wave frequencies.
\end{abstract}

Keywords Floating systems · Free-surface flow · Ship motion · Source function · Stratified fluid · Unsteady flow · Wave motion

\section{Background}

Two-layer fluids are normally used to model certain types of density stratification in the ocean. Density stratification is quite common and is mainly caused by variation of salinity and water temperature with depth. Very often the change in density is confined within a thin pycnocline, above and below which, the water is well mixed, and the density is practically constant. For marine vehicles operating in this environment, a two-layer fluid is a good model to study the effects of density stratification on vehicle performance. In this paper, the density in each layer is taken as constant, and the fluid is assumed to be inviscid, incompressible and the flow irrotational. The pycnocline is assumed to be infinitesimally thin, and there is a density discontinuity across the interface between the upper and lower fluid

T. C. Nguyen

Naval Surface Warfare Center, Panama City, FL 32407, USA

R. W. Yeung $(\varangle)$

Ocean Engineering Group, Department of Mechanical Engineering, University of California at Berkeley,

Berkeley, CA 94720-1740, USA

e-mail: rwyeung@berkeley.edu 
layers. The density difference for these pycnoclines is usually very small. It is represented by the non-dimensional parameter $\varepsilon=\left(\rho_{2}-\rho_{1}\right) / \rho_{2}$ where $\rho_{1}$ and $\rho_{2}$ are the densities of the upper and lower fluid, respectively. In fjords, pycnoclines with $\varepsilon$ on the order of $10^{-3}$ to $10^{-2}$ are typical. Despite the small density difference, internal waves generated at the fluid interface can cause a loss in ship speed and maneuverability when the ship is moving slowly. This effect on ship motion is commonly known as the "dead-water" phenomenon and was first documented by Fridjof Nansen who had encountered it several times during his journey to the north pole. The large increase in ship resistance was correctly attributed to the generation of internal waves by Bjerknes and confirmed in a towing tank by his student Ekman [1]. Ekman noted wave drag was largest when the ship speed approached the maximum phase speed of the internal waves.

Theoretical investigations of internal wave resistance using a two-layer fluid began with the works of Sretenskii [2] and Hudimac [3], who independently derived the Green functions for a steady source in the upper fluid layer. Both assumed the lower fluid to be infinitely deep and derived the wave-resistance formulas for a thin ship that included effects of both surface and internal waves. These formulas are generalizations of the well-known Michell integral [4] for the wave resistance in a single fluid. Sabuncu [5] considered a steadily translating source for other geometric variations. These include both fluid layers of infinite depths and fluid bounded on top and bottom by rigid, horizontal planes. One important case that was not addressed in [5] was the finite-depth case where the upper fluid is bounded by the free surface, and the lower fluid is bounded by a rigid bottom. Such source functions are used to study the wave resistance of ships moving in harbors or channels where the water depths are finite but at speeds high enough so that free-surface effects cannot be neglected. Uspenskii [6] obtained the wave components of the Green functions but not the complete expressions necessary for use in boundary-integral-equation methods. Miloh et al. [7] derived the complete Green functions with the assumption of small density difference, $\varepsilon \ll 1$. Nguyen and Yeung [8] removed this restriction and gave the complete Green functions for arbitrary $\varepsilon$. Surface-wave and internal-wave patterns associated with steady two-layer flow were examined by Yeung and Nguyen [9].

Unlike the steadily translating source, the time-dependent sources for a two-layer fluid have not been well studied. Most investigations have focused on the two-dimensional case where infinite cylinders are modeled and useful results on wave reflection and transmission were obtained [10-15]. Sturova and Syui [16] computed the added-mass and damping coefficients for cylinders with Lewis-form cross-sections that pierce the fluid interface. However, for practical applications, three-dimensional sources are often needed to analyze more complex structures. In a single fluid, three-dimensional, unsteady sources are widely used to compute the motions of ships and offshore platforms in waves. For a frequency-domain formulation, the oscillating source with or without forward speed was used [17, 18]. For a time-domain solution, King et al. [19] and Lin and Yue [20] employed the source with a delta-function strength. This source can be obtained in a straight-forward way from the more general transient source of arbitrary strength and motion.

In this paper, we derive the analogous three-dimensional sources for a two-layer fluid of finite depth. The upper fluid is bounded above by the usual free surface and the lower fluid is bounded below by a rigid, flat bottom. The density difference between the two layers is finite. By not assuming a small density difference, the Green functions given here can also be used to investigate the effect of a bottom layer of fluid mud on the ship's added masses and damping coefficients. Although the kinematic viscosity $\nu_{2}$ of the fluid mud can be many times larger than that of water, Zilman and Miloh [21] have shown that the hydrodynamic coefficients are weakly dependent on the mud viscosity. Thus, our results, which neglect viscosity in both layers, are applicable to this problem. The sources are restricted to the upper fluid layer. This restriction, however, is not very limiting since the drafts of most vessels are small enough to be above the pycnocline or the mud layer. The paper is organized as follows. In Sect. 2, the velocity potentials for a transient source with arbitrary strength and motion in three dimensions are derived. They are used as the starting point for obtaining the potentials of a stationary, oscillating source in Sect. 3 and the potentials of a translating, oscillating source in Sect. 4. With the expressions for the sources given here, existing ship-motion computer codes can be easily modified to evaluate the effects of density stratification and internal waves on ship motions. Section 5 presents a sample application of the stationary, oscillating source to solve the radiation and diffraction problem of a floating rectangular barge. The barge motions induced by either surface or internal waves are presented. 


\section{Velocity potentials of a transient source}

For the most general situation, we begin our derivation with a source of variable strength, starting from rest and moving along an arbitrary path in the upper fluid layer. This transient source is the most general source considered here. Other sources commonly used in ship-motion analyses can be obtained from this source by specifying the appropriate strength and motion. Consider a fixed, rectangular coordinate system $O x y z$ where the $x y$-plane coincides with the undisturbed interface between the two fluid layers, and the positive $z$-axis points upwards. The densities and depths of the upper and lower fluid layer are indicated by $\rho_{1}, h_{1}$ and $\rho_{2}, h_{2}$, respectively, as shown in Fig. 1 . For a source located at a point $\vec{\xi}=(\xi, \eta, \zeta)$, the velocity potentials of the induced flows in the upper and lower layers are designated by $\Phi^{(1)}$ and $\Phi^{(2)}$, respectively.

The governing equations for $\Phi^{(m)}, m=1,2$, are

$\nabla^{2} \Phi^{(1)}=\delta(x-\xi, y-\eta, z-\zeta), \quad 0<z<h_{1}$,

$\nabla^{2} \Phi^{(2)}=0, \quad-h_{2}<z<0$.

The time-dependent linearized boundary condition on the free surface is

$\Phi_{t t}^{(1)}+g \Phi_{z}^{(1)}=0, \quad z=h_{1}, \quad t \geq 0$,

and the conditions on the interface are

$\Phi_{z}^{(1)}=\Phi_{z}^{(2)}, \quad z=0, \quad t \geq 0$,

$\gamma\left(\Phi_{t t}^{(1)}+g \Phi_{z}^{(1)}\right)=\Phi_{t t}^{(2)}+g \Phi_{z}^{(2)}, \quad z=0, \quad t \geq 0$,

where $g$ is the gravitational acceleration, and $\gamma=\rho_{1} / \rho_{2}$ is the density ratio. At the rigid bottom, the no-flux boundary condition applies,

$\Phi_{z}^{(2)}=0, \quad z=-h_{2}, \quad t \geq 0$,

and far away from the source, we require the velocity to vanish,

$\lim _{R \rightarrow \infty} \nabla \Phi^{(m)}=0, \quad t \geq 0$,

where $R^{2}=[x-\xi(t)]^{2}+[y-\eta(t)]^{2}$. Finally, the initial conditions for $\Phi^{(m)}$ in an initially quiescent flow are:

$\Phi^{(1)}=\Phi_{t}^{(1)}=0, \quad z=0, h_{1}, \quad t=0$,

$\Phi^{(2)}=\Phi_{t}^{(2)}=0, \quad z=0, \quad t=0$.

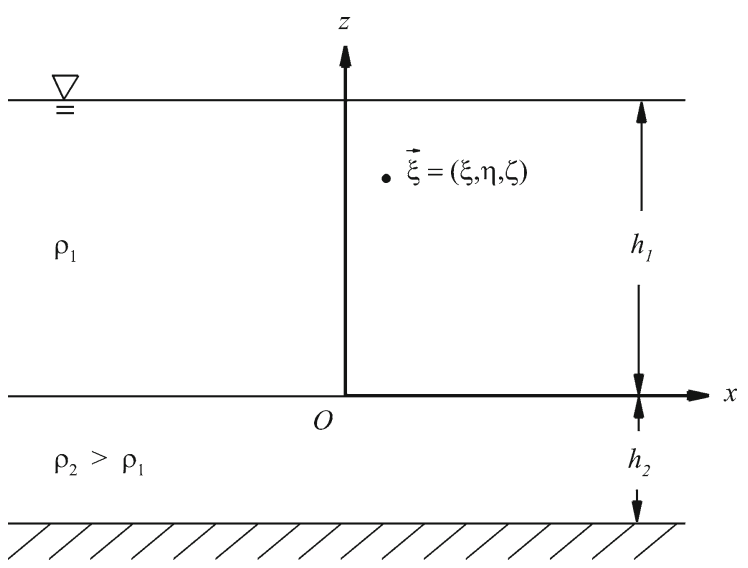

Fig. 1 Coordinate system for flows in a two-layer fluid of finite depth 
We assume the solution to the above initial, boundary-value problem to have the following forms:

$\Phi^{(1)}=v(t) \sum_{n=-\infty}^{\infty}\left(\frac{1}{r_{n}}-\frac{1}{r_{1 n}}\right)+\phi^{(1)}$ and $\Phi^{(2)}=\phi^{(2)}$,

where $v(t)$ is the source strength and

$r_{n}^{2}=R^{2}+\left(z-\zeta-2 n h_{1}\right)^{2}$ and $r_{1 n}^{2}=R^{2}+\left(z+\zeta-2 n h_{1}\right)^{2}$.

where the image series are constructed to satisfy homogeneous conditions on $z=0$ and $z=h_{1}$. The governing equation for $\phi^{(m)}$ is now simply the Laplace equation:

$\nabla^{2} \phi^{(m)}=0$

and the initial conditions, obtained by inserting (10) into (8) and (9), are

$\phi^{(1)}=\phi_{t}^{(1)}=0, \quad z=0, h_{1}, \quad t=0$,

$\phi^{(2)}=\phi_{t}^{(2)}=0, \quad z=0, \quad t=0$,

To solve for $\phi^{(m)}$, we introduce the following Laplace and Fourier transforms in time and space:

$$
\begin{aligned}
& \bar{\phi}^{(m)}(x, y, z, s)=L\left\{\phi^{(m)}(x, y, z, t)\right\}=\int_{0}^{\infty} \phi^{(m)}(x, y, z, t) \mathrm{e}^{-s t} \mathrm{~d} t, \\
& \tilde{\phi}^{(m)}\left(k_{1}, k_{2}, z, s\right)=F_{x y}\left\{\bar{\phi}^{(m)}(x, y, z, s)\right\}=\frac{1}{2 \pi} \int_{-\infty}^{\infty} \int_{-\infty}^{\infty} \bar{\phi}^{(m)}(x, y, z, s) \mathrm{e}^{-\mathrm{i}\left(k_{1} x+k_{2} y\right)} \mathrm{d} x \mathrm{~d} y, \\
& \bar{\phi}^{(m)}(x, y, z, s)=F_{x y}^{-1}\left\{\tilde{\phi}^{(m)}\left(k_{1}, k_{2}, z, s\right)\right\}=\frac{1}{2 \pi} \int_{-\infty}^{\infty} \int_{-\infty}^{\infty} \tilde{\phi}^{(m)}\left(k_{1}, k_{2}, z, s\right) \mathrm{e}^{\mathrm{i}\left(k_{1} x+k_{2} y\right)} \mathrm{d} k_{1} \mathrm{~d} k_{2} .
\end{aligned}
$$

Application of these transforms to (12) yields the following equation for $\tilde{\phi}^{(m)}$ :

$$
-k^{2} \tilde{\phi}^{(m)}+\tilde{\phi}_{z z}^{(m)}=0
$$

where $k^{2}=k_{1}^{2}+k_{2}^{2}$. The solutions of (18) can be written as:

$$
\begin{aligned}
& \tilde{\phi}^{(1)}\left(k_{1}, k_{2}, z, s\right)=\tilde{A}\left(k_{1}, k_{2}, s\right) \mathrm{e}^{k z}+\tilde{B}\left(k_{1}, k_{2}, s\right) \mathrm{e}^{-k z}, \\
& \tilde{\phi}^{(2)}\left(k_{1}, k_{2}, z, s\right)=\tilde{C}\left(k_{1}, k_{2}, s\right) \cosh \left[k\left(z+h_{2}\right)\right],
\end{aligned}
$$

where the no-flux, bottom condition in (6) has been used to obtain (20). The unknown coefficients $\tilde{A}, \tilde{B}$, and $\tilde{C}$ can be determined by applying the remaining boundary conditions on the free surface and interface, viz. Eqs. 3-5. Inserting $\Phi^{(1)}$ as given by (10) into the free-surface condition, Eq. 3, we obtain:

$\phi_{t t}^{(1)}+g \phi_{z}^{(1)}+g v(t) \sum_{n=-\infty}^{\infty} \frac{\partial}{\partial z}\left(\frac{1}{r_{n}}-\frac{1}{r_{1 n}}\right)=0, \quad z=h_{1}$.

Taking the Laplace transform of (21) and applying the initial condition (13), we arrive at:

$$
s^{2} \bar{\phi}^{(1)}+g \bar{\phi}_{z}^{(1)}+g \int_{0}^{\infty}\left[v(t) \sum_{n=-\infty}^{\infty} \frac{\partial}{\partial z}\left(\frac{1}{r_{n}}-\frac{1}{r_{1 n}}\right)\right] \mathrm{e}^{-s t} \mathrm{~d} t=0, \quad z=h_{1} .
$$


2.1 Determination of the Fourier coefficients $\tilde{A}, \tilde{B}$, and $\tilde{C}$

To obtain the expressions for the $\tilde{A}, \tilde{B}$, and $\tilde{C}$, we can first write $\bar{\phi}^{(1)}$ in terms of $\tilde{A}$ and $\tilde{B}$ so that (22) will provide a relation involving these two coefficients. Using the definition of the inverse Fourier transform in (17) and solution for $\tilde{\phi}^{(1)}$ in (19), we obtain:

$\bar{\phi}^{(1)}\left(x, y, h_{1}, s\right)=\frac{1}{2 \pi} \int_{-\infty}^{\infty} \int_{-\infty}^{\infty}\left(\tilde{A} \mathrm{e}^{k h_{1}}+\tilde{B} \mathrm{e}^{-k h_{1}}\right) \mathrm{e}^{\mathrm{i}\left(k_{1} x+k_{2} y\right)} \mathrm{d} k_{1} \mathrm{~d} k_{2}$.

From the well-known representation of $1 / r$ (see [22, 6.611 and 3.937]):

$\frac{1}{\sqrt{x^{2}+y^{2}+z^{2}}}=\frac{1}{2 \pi} \int_{-\infty}^{\infty} \int_{-\infty}^{\infty} \frac{\mathrm{e}^{-k|z|}}{k} \mathrm{e}^{\mathrm{i}\left(k_{1} x+k_{2} y\right)} \mathrm{d} k_{1} \mathrm{~d} k_{2}$

we can write

$$
\begin{aligned}
& 1 / r_{n}=\frac{1}{2 \pi} \int_{-\infty}^{\infty} \int_{-\infty}^{\infty} \mathrm{e}^{-k\left|z-\zeta-2 n h_{1}\right|} \frac{\mathrm{e}^{\mathrm{i}\left[k_{1}(x-\xi)+k_{2}(y-\eta)\right]}}{k} \mathrm{~d} k_{1} \mathrm{~d} k_{2}, \\
& 1 / r_{1 n}=\frac{1}{2 \pi} \int_{-\infty}^{\infty} \int_{-\infty}^{\infty} \mathrm{e}^{-k\left|z+\zeta-2 n h_{1}\right|} \frac{\mathrm{e}^{\mathrm{i}\left[k_{1}(x-\xi)+k_{2}(y-\eta)\right]}}{k} \mathrm{~d} k_{1} \mathrm{~d} k_{2},
\end{aligned}
$$

which lead to:

$$
\begin{aligned}
& \frac{\partial}{\partial z}\left(1 / r_{n}\right)_{z=h_{1}}=\frac{\operatorname{sgn}(n-1)}{2 \pi} \int_{-\infty}^{\infty} \int_{-\infty}^{\infty} \mathrm{e}^{-k\left|\zeta+(2 n-1) h_{1}\right|} \mathrm{e}^{\mathrm{i}\left[k_{1}(x-\xi)+k_{2}(y-\eta)\right]} \mathrm{d} k_{1} \mathrm{~d} k_{2}, \\
& \frac{\partial}{\partial z}\left(1 / r_{1 n}\right)_{z=h_{1}}=\frac{\operatorname{sgn}(n-1)}{2 \pi} \int_{-\infty}^{\infty} \int_{-\infty}^{\infty} \mathrm{e}^{-k\left|-\zeta+(2 n-1) h_{1}\right|} \mathrm{e}^{\mathrm{i}\left[k_{1}(x-\xi)+k_{2}(y-\eta)\right]} \mathrm{d} k_{1} \mathrm{~d} k_{2},
\end{aligned}
$$

where $\operatorname{sgn}(n)=1$ if $n \geq 0$ and $\operatorname{sgn}(n)=-1$ if $n<0$. Finally, substituting (23), (27), and (28) in (22), we obtain the following equation relating the coefficients $\tilde{A}$ and $\tilde{B}$ :

$\left(s^{2}+g k\right) \tilde{A} \mathrm{e}^{k h_{1}}+\left(s^{2}-g k\right) \tilde{B} \mathrm{e}^{-k h_{1}}=\frac{2 g}{\sinh k h_{1}} \int_{0}^{\infty} v(t) \mathrm{e}^{-\mathrm{i}\left[k_{1} \xi(t)+k_{2} \eta(t)\right]} \sinh [k \zeta(t)] \mathrm{e}^{-s t} \mathrm{~d} t$.

In deriving the above equation, use has been made of the following identity, given in [23], to eliminate the infinite series in (22):

$\frac{\mathrm{e}^{-k h_{1}}}{\sinh k h_{1}}=2 \sum_{n=1}^{\infty} \mathrm{e}^{-2 n k h_{1}}$.

Applying the same procedures to the interface kinematic and dynamic boundary conditions, Eqs. 4 and 5, we obtain two additional equations for the unknown coefficients $\tilde{A}, \tilde{B}$, and $\tilde{C}$ :

$\tilde{A}-\tilde{B}-\tilde{C} \sinh k h_{2}=\frac{2}{k \sinh k h_{1}} \int_{0}^{\infty} v(t) \mathrm{e}^{-\mathrm{i}\left[k_{1} \xi(t)+k_{2} \eta(t)\right]} \sinh \left[k\left(\zeta(t)-h_{1}\right)\right] \mathrm{e}^{-s t} \mathrm{~d} t$,

$\gamma s^{2} \tilde{A}+\gamma s^{2} \tilde{B}-\left(s^{2} \cosh k h_{2}+\varepsilon g k \sinh k h_{2}\right) \tilde{C}=0$.

Equations 29, 31, and 32 now form a system of three linear equations for $\tilde{A}, \tilde{B}$, and $\tilde{C}$ which can readily be solved. Once these coefficients are obtained, the inverse Fourier transform of $\tilde{\phi}^{(m)}$ can be performed to give $\bar{\phi}^{(m)}$, and the inverse Laplace transform can then be applied to give an expression for $\phi^{(m)}$. The inversion process is described in detail in Sect. 2.2 for the potential $\tilde{\phi}^{(2)}$ since $\tilde{\phi}^{(2)}$ is simpler than $\tilde{\phi}^{(1)}$, but the steps are essentially the same for both potentials. 
2.2 Inversion and final source forms

The solution of the system of linear equations, (29), (31) and (32), yields the following expression for $\tilde{C}$ :

$$
\begin{aligned}
\tilde{C}\left(k_{1}, k_{2}, s\right)= & \tilde{C}_{1}(k, s) \int_{0}^{\infty} v(t) \mathrm{e}^{-\mathrm{i}\left[k_{1} \xi(t)+k_{2} \eta(t)\right]} \sinh [k \zeta(t)] \mathrm{e}^{-s t} \mathrm{~d} t \\
& +\left[\tilde{C}_{0}(k)+\tilde{C}_{2}(k, s)\right] \int_{0}^{\infty} v(t) \mathrm{e}^{-\mathrm{i}\left[k_{1} \xi(t)+k_{2} \eta(t)\right]} \sinh \left[k\left(\zeta(t)-h_{1}\right)\right] \mathrm{e}^{-s t} \mathrm{~d} t
\end{aligned}
$$

where $\tilde{C}_{0}(k), \tilde{C}_{1}(k, s)$, and $\tilde{C}_{2}(k, s)$ are given by:

$$
\begin{aligned}
& \tilde{C}_{0}=\frac{-2 \gamma}{k Q(k)}, \quad \tilde{C}_{1}=\frac{2 g \gamma s^{2}}{\sinh \left(k h_{1}\right) Q(k)\left(s^{2}+\omega_{1}^{2}\right)\left(s^{2}+\omega_{2}^{2}\right)}, \\
& \tilde{C}_{2}=\frac{2 \gamma\left[\sinh k h_{1}\left(\omega_{1}^{2}+\omega_{2}^{2}\right)-g k \cosh k h_{1}\right] s^{2}+2 \gamma \sinh k h_{1} \omega_{1}^{2} \omega_{2}^{2}}{k \sinh \left(k h_{1}\right) Q(k)\left(s^{2}+\omega_{1}^{2}\right)\left(s^{2}+\omega_{2}^{2}\right)}, \\
& Q(k)=\cosh k h_{1} \cosh k h_{2}+\gamma \sinh k h_{1} \sinh k h_{2}, \\
& \omega_{n}^{2}(k)=\frac{g k}{2}\left[\frac{\left(t_{1}+t_{2}\right)+(-1)^{n+1} \sqrt{\left(t_{1}+t_{2}\right)^{2}-4 \varepsilon t_{1} t_{2}\left(1+\gamma t_{1} t_{2}\right)}}{1+\gamma t_{1} t_{2}}\right],
\end{aligned}
$$

with the notation $t_{1}=\tanh \left(k h_{1}\right), t_{2}=\tanh \left(k h_{2}\right)$ being used for simplicity. Taking the inverse Fourier transform of $\tilde{\phi}^{(2)}$, we have:

$\bar{\phi}^{(2)}(x, y, z, s)=\frac{1}{2 \pi} \int_{-\infty}^{\infty} \int_{-\infty}^{\infty} \tilde{C}\left(k_{1}, k_{2}, s\right) \cosh \left[k\left(z+h_{2}\right)\right] \mathrm{e}^{\mathrm{i}\left(k_{1} x+k_{2} y\right)} \mathrm{d} k_{1} \mathrm{~d} k_{2}$.

The expression of (33) can now be inserted into (38). The results will be simpler if the following "polar representations" are introduced:

$$
\begin{aligned}
& k_{1}+i k_{2}=k \mathrm{e}^{\mathrm{i} \theta}, \\
& R=\sqrt{[x-\xi(t)]^{2}+[y-\eta(t)]^{2}}, \\
& \omega=[x-\xi(t)] \cos \theta+[y-\eta(t)] \sin \theta .
\end{aligned}
$$

Further, if the following integral representation of the Bessel function $J_{o}$ is used [24, p. 491]:

$$
J_{0}(k R(t))=\frac{1}{2 \pi} \int_{-\pi}^{\pi} \mathrm{e}^{\mathrm{i} k((x-\xi(t)) \cos \theta+(y-\eta(t)) \sin \theta)} \mathrm{d} \theta=\frac{1}{2 \pi} \int_{-\pi}^{\pi} \mathrm{e}^{\mathrm{i} k \omega} \mathrm{d} \theta,
$$

the potential $\bar{\phi}^{(2)}(x, y, z, s)$ becomes:

$$
\begin{aligned}
\bar{\phi}^{(2)}= & \int_{0}^{\infty} k \tilde{C}_{1} \cosh \left[k\left(z+h_{2}\right)\right] \int_{0}^{\infty} v(t) \sinh [k \zeta(t)] J_{0}(k R(t)) \mathrm{e}^{-s t} \mathrm{~d} t \mathrm{~d} k \\
& +\int_{0}^{\infty} k\left(\tilde{C}_{0}+\tilde{C}_{2}\right) \cosh \left[k\left(z+h_{2}\right)\right] \int_{0}^{\infty} v(t) \sinh \left[k\left(\zeta(t)-h_{1}\right)\right] J_{0}(k R(t)) \mathrm{e}^{-s t} \mathrm{~d} t \mathrm{~d} k .
\end{aligned}
$$


The time integrals of (43) involving $\tilde{C}_{1}$ and $\tilde{C}_{2}$ can be rewritten using the convolution theorem, namely,

$$
\begin{aligned}
& \tilde{C}_{j}(k, s) \int_{0}^{\infty} v(t) \sinh \left[k\left(\zeta(t)-(j-1) h_{1}\right)\right] J_{0}(k R(t)) \mathrm{e}^{-s t} \mathrm{~d} t \\
& \quad=L\left\{\int_{0}^{t} \tilde{c}_{j}(k, t-\tau) v(\tau) \sinh \left[k\left(\zeta(\tau)-(j-1) h_{1}\right)\right] J_{0}(k R(\tau)) \mathrm{d} \tau\right\}, \quad j=1,2,
\end{aligned}
$$

where

$$
\begin{aligned}
& \tilde{c}_{1}(k, t)=L^{-1}\left\{\tilde{C}_{1}(k, s)\right\}=\sum_{n=1}^{2} \frac{(-1)^{n+1} 2 g \gamma \omega_{n} \sin \omega_{n} t}{\sinh k h_{1} Q(k)\left(\omega_{1}^{2}-\omega_{2}^{2}\right)}, \\
& \tilde{c}_{2}(k, t)=L^{-1}\left\{\tilde{C}_{2}(k, s)\right\}=\sum_{n=1}^{2} \frac{(-1)^{n+1} 2 \gamma \omega_{n}\left(\omega_{n}^{2} \sinh k h_{1}-g k \cosh k h_{1}\right) \sin \omega_{n} t}{k \sinh k h_{1} Q(k)\left(\omega_{1}^{2}-\omega_{2}^{2}\right)} .
\end{aligned}
$$

Since $\tilde{C}_{0}(k)$ is not a function of $s$, it is not necessary to apply the convolution theorem to the product containing this term. With the use of these results, Eq. 43 becomes:

$$
\begin{aligned}
\bar{\phi}^{(2)}= & \int_{0}^{\infty} k \tilde{C}_{0} \cosh \left(k\left(z+h_{2}\right)\right) \cdot L\left\{v(t) \sinh \left(k\left(\zeta(t)-h_{1}\right)\right) J_{0}(k R(t))\right\} \mathrm{d} k \\
& +\int_{0}^{\infty} k \cosh \left(k\left(z+h_{2}\right)\right) \cdot L\left\{\int_{0}^{t} \tilde{c}_{1}(k, t-\tau) v(\tau) \sinh (k \zeta(\tau)) J_{0}(k R(\tau)) d \tau\right\} \mathrm{d} k \\
& +\int_{0}^{\infty} k \cosh \left(k\left(z+h_{2}\right)\right) \cdot L\left\{\int_{0}^{t} \tilde{c}_{2}(k, t-\tau) v(\tau) \sinh \left(k\left(\zeta(\tau)-h_{1}\right)\right) J_{0}(k R(\tau)) d \tau\right\} \mathrm{d} k .
\end{aligned}
$$

Taking the inverse Laplace transform of the above equation, we obtain the following:

$$
\begin{aligned}
\phi^{(2)}= & v(t) \int_{0}^{\infty} k \tilde{C}_{0}(k) \cosh \left(k\left(z+h_{2}\right)\right) \sinh \left(k\left(\zeta(t)-h_{1}\right)\right) J_{0}(k R(t)) \mathrm{d} k \\
& +\int_{0}^{\infty} k \cosh \left(k\left(z+h_{2}\right)\right) \int_{0}^{t} \tilde{c}_{1}(k, t-\tau) v(\tau) \sinh (k \zeta(\tau)) J_{0}(k R(\tau)) \mathrm{d} \tau \mathrm{d} k \\
& +\int_{0}^{\infty} k \cosh \left(k\left(z+h_{2}\right)\right) \int_{0}^{t} \tilde{c}_{2}(k, t-\tau) v(\tau) \sinh \left(k\left(\zeta(\tau)-h_{1}\right)\right) J_{0}(k R(\tau)) \mathrm{d} \tau \mathrm{d} k .
\end{aligned}
$$

Substituting Eqs. 34, 45, 46 for $\tilde{C}_{0}, \tilde{c}_{1}$, and $\tilde{c}_{2}$, we finally obtain the following equation for $\Phi^{(2)}(x, y, z, t)$ :

$$
\begin{aligned}
\Phi^{(2)}=\phi^{(2)}= & v(t) \int_{0}^{\infty} \frac{-2 \gamma \cosh \left(k\left(z+h_{2}\right)\right) \sinh \left(k\left(\zeta(t)-h_{1}\right)\right) J_{0}(k R(t))}{\cosh k h_{1} \cosh k h_{2}+\gamma \sinh k h_{1} \sinh k h_{2}} \mathrm{~d} k \\
& +\sum_{n=1}^{2} \int_{0}^{\infty} \frac{2 g k \omega_{n}}{\sinh k h_{1}\left(\cosh k h_{1} \cosh k h_{2}+\gamma \sinh k h_{1} \sinh k h_{2}\right)\left(\omega_{1}^{2}-\omega_{2}^{2}\right)} \\
& \times \int_{0}^{t} v(\tau) P_{n}^{(2)}(k, z, \zeta(\tau)) \sin \left(\omega_{n}(t-\tau)\right) J_{0}(k R(\tau)) \mathrm{d} \tau \mathrm{d} k,
\end{aligned}
$$


where

$P_{n}^{(2)}=(-1)^{n+1} \gamma \cosh \left(k\left(z+h_{2}\right)\right)\left(\sinh k \zeta+\left(\frac{\omega_{n}^{2}}{g k} \sinh k h_{1}-\cosh k h_{1}\right) \sinh \left(k\left(\zeta-h_{1}\right)\right)\right)$.

The functions $\omega_{1}(k)$ and $\omega_{2}(k)$ are the two dispersion relations corresponding to the "surface-wave mode" and the "internal-wave mode", respectively. We will elaborate on these two possible wave modes later when we derive the velocity potentials of a periodic, progressive wave in a two-layer fluid of finite depth.

Applying the same inversion procedures to the potential $\tilde{\phi}^{(1)}\left(k_{1}, k_{2}, z, s\right)$, we obtain an analogous equation for $\Phi^{(1)}(x, y, z, t)$ :

$$
\begin{aligned}
\Phi^{(1)}= & v(t) \sum_{n=-\infty}^{\infty}\left(\frac{1}{r_{n}}-\frac{1}{r_{1 n}}\right) \\
& +v(t) \int_{0}^{\infty} \frac{2 \cosh k h_{2} \sinh \left(k\left(z-h_{1}\right)\right) \sinh \left(k\left(\zeta(t)-h_{1}\right)\right) J_{0}(k R(t))}{\sinh k h_{1}\left(\cosh k h_{1} \cosh k h_{2}+\gamma \sinh k h_{1} \sinh k h_{2}\right)} \mathrm{d} k \\
& +\sum_{n=1}^{2} \int_{0}^{\infty} \frac{2 g k \omega_{n}}{\sinh k h_{1}\left(\cosh k h_{1} \cosh k h_{2}+\gamma \sinh k h_{1} \sinh k h_{2}\right)\left(\omega_{1}^{2}-\omega_{2}^{2}\right)} \\
& \times \int_{0}^{t} v(\tau) P_{n}^{(1)}(k, z, \zeta(\tau)) \sin \left(\omega_{n}(t-\tau)\right) J_{0}(k R(\tau)) \mathrm{d} \tau \mathrm{d} k,
\end{aligned}
$$

where

$$
\begin{aligned}
P_{n}^{(1)}= & (-1)^{n+1}\left\{\gamma \sinh k h_{2} \sinh k z \sinh k \zeta+\left(\cosh k h_{2}-\frac{\varepsilon g k}{\omega_{n}^{2}} \sinh k h_{2}\right)\right. \\
& \left.\times\left[\cosh k z \sinh k \zeta-\cosh \left(k\left(z-h_{1}\right)\right) \sinh \left(k\left(\zeta-h_{1}\right)\right)-\frac{\omega_{n}^{2}}{g k} \sinh \left(k\left(z-h_{1}\right)\right) \sinh \left(k\left(\zeta-h_{1}\right)\right)\right]\right\} .
\end{aligned}
$$

The equations for $\Phi^{(1)}$ and $\Phi^{(2)}$ have forms similar to the transient potentials for a single fluid. In fact, it is possible to obtain the potentials for a single fluid of finite and infinite depth from either $\Phi^{(1)}$ or $\Phi^{(2)}$. If we set $\gamma=1$ in either (49) or (51), we will recover the potential for a single fluid of finite depth and, if we also let $h_{2} \rightarrow \infty$, we will obtain the potential for a single fluid of infinite depth.

\section{Velocity potentials of a stationary, oscillating source}

The stationary source with oscillating strength is mainly used in boundary-integral methods to compute the motions of floating systems and offshore structures in waves. The source potentials, or Green functions $G^{(m)}$, can be obtained directly by solving a boundary-value problem in the frequency domain or indirectly from the potentials $\Phi^{(m)}$ of a transient source. Since we have already derived the equations for $\Phi^{(m)}$, it is simpler to start our development with these equations. After fixing the source location at point $(\xi, \eta, \zeta)$ and setting the source strength $v(t)=\cos \sigma t$ in (49) and (51), we can take the limit as $t \rightarrow \infty$ to obtain the desired Green functions. This procedure is elaborated below for the potential $G^{(1)}$ in the upper fluid layer. The same procedures can be applied to obtain $G^{(2)}$. Note that this procedure automatically yields outgoing waves and no radiation condition is needed.

Substituting $v(t)=\cos \sigma t$ in (51) and noting that $(\xi, \eta, \zeta)$ is now no longer a function of time, we obtain:

$$
\begin{aligned}
& G^{(1)}=\cos \sigma t \sum_{n=-\infty}^{\infty}\left(\frac{1}{r_{n}}-\frac{1}{r_{1 n}}\right)+\cos \sigma t \int_{0}^{\infty} \frac{2 \cosh k h_{2} \sinh \left(k\left(z-h_{1}\right)\right) \sinh \left(k\left(\zeta-h_{1}\right)\right) J_{0}(k R)}{\sinh k h_{1}\left(\cosh k h_{1} \cosh k h_{2}+\gamma \sinh k h_{1} \sinh k h_{2}\right)} \mathrm{d} k \\
& +\sum_{n=1}^{2} \int_{0}^{\infty} \frac{2 g k \omega_{n} P_{n}^{(1)}(k, z, \zeta) J_{0}(k R)}{\sinh k h_{1}\left(\cosh k h_{1} \cosh k h_{2}+\gamma \sinh k h_{1} \sinh k h_{2}\right)\left(\omega_{1}^{2}-\omega_{2}^{2}\right)} \int_{0}^{t} \cos \sigma \tau \sin \left(\omega_{n}(t-\tau)\right) \mathrm{d} \tau \mathrm{d} k .
\end{aligned}
$$


Performing the integration with respect to $\tau$ in the last integral, we have:

$$
\int_{0}^{t} \cos \sigma \tau \sin \left(\omega_{n}(t-\tau)\right) \mathrm{d} \tau=\cos \sigma t\left(\frac{\omega_{n}}{\omega_{n}^{2}-\sigma^{2}}\right)-\cos \omega_{n} t\left(\frac{\omega_{n}}{\omega_{n}^{2}-\sigma^{2}}\right) .
$$

Using this result in (53), we can write:

$$
\begin{aligned}
G^{(1)}= & \cos \sigma t \sum_{n=-\infty}^{\infty}\left(\frac{1}{r_{n}}-\frac{1}{r_{1 n}}\right)+\cos \sigma t \int_{0}^{\infty} \frac{2 \cosh k h_{2} \sinh \left(k\left(z-h_{1}\right)\right) \sinh \left(k\left(\zeta-h_{1}\right)\right) J_{0}(k R)}{\sinh k h_{1}\left(\cosh k h_{1} \cosh k h_{2}+\gamma \sinh k h_{1} \sinh k h_{2}\right)} \mathrm{d} k \\
& +\cos \sigma t \sum_{n=1}^{2} \mathrm{pv} \int_{0}^{\infty} \frac{2 g k \omega_{n}^{2} P_{n}^{(1)}(k, z, \zeta) J_{0}(k R)}{\sinh k h_{1}\left(\cosh k h_{1} \cosh k h_{2}+\gamma \sinh k h_{1} \sinh k h_{2}\right)\left(\omega_{1}^{2}-\omega_{2}^{2}\right)\left(\omega_{n}^{2}-\sigma^{2}\right)} \mathrm{d} k \\
& -\sum_{n=1}^{2} \mathrm{pv} \int_{0}^{\infty} \frac{2 g k \omega_{n}^{2} P_{n}^{(1)}(k, z, \zeta) J_{0}(k R) \cos \omega_{n} t}{\sinh k h_{1}\left(\cosh k h_{1} \cosh k h_{2}+\gamma \sinh k h_{1} \sinh k h_{2}\right)\left(\omega_{1}^{2}-\omega_{2}^{2}\right)\left(\omega_{n}^{2}-\sigma^{2}\right)} \mathrm{d} k .
\end{aligned}
$$

The integrals in the last two terms contain simple poles at $k=k_{n}$ where $k_{n}$ are given implicitly by $\omega_{n}^{2}\left(k_{n}\right)=\sigma^{2}$. The notation "pv" indicates that the principal value is to be taken. Next, we need to obtain the limit of $G^{(1)}$ as $t \rightarrow \infty$. The first three terms of (55) pose no difficulties. The last term, however, must be manipulated slightly before we can take the limit. Using the addition formula for cosine, we can write

$\cos \omega_{n} t=\cos \left(\left(\omega_{n}-\sigma\right) t+\sigma t\right)=\cos \left(\left(\omega_{n}-\sigma\right) t\right) \cos \sigma t-\sin \left(\left(\omega_{n}-\sigma\right) t\right) \sin \sigma t$,

while the last integral of (55) becomes

$$
-\cos \sigma t \sum_{n=1}^{2} \mathrm{pv} \int_{0}^{\infty} \frac{F_{n}(k) \cos \left(\left(\omega_{n}-\sigma\right) t\right)}{\omega_{n}-\sigma} \mathrm{d} k+\sin \sigma t \sum_{n=1}^{2} \mathrm{pv} \int_{0}^{\infty} \frac{F_{n}(k) \sin \left(\left(\omega_{n}-\sigma\right) t\right)}{\omega_{n}-\sigma} \mathrm{d} k,
$$

with

$$
F_{n}(k)=\frac{2 g k \omega_{n}^{2} P_{n}^{(1)}(k, z, \zeta) J_{0}(k R)}{\sinh k h_{1}\left(\cosh k h_{1} \cosh k h_{2}+\gamma \sinh k h_{1} \sinh k h_{2}\right)\left(\omega_{1}^{2}-\omega_{2}^{2}\right)\left(\omega_{n}+\sigma\right)} .
$$

To obtain the limit as $t \rightarrow \infty$ of (57), we use the following formulas from the Fourier Integral Theorem [24, p. 493] which show that for a function $f(k)$ with a simple zero at $k=k_{1}$ :

$$
\begin{aligned}
& \lim _{t \rightarrow \infty} \mathrm{pv} \int_{0}^{\infty} \frac{F(k) \cos (t f(k))}{f(k)} \mathrm{d} k=O\left(\frac{1}{t}\right), \\
& \lim _{t \rightarrow \infty} \mathrm{pv} \int_{0}^{\infty} \frac{F(k) \sin (t f(k))}{f(k)} \mathrm{d} k=\frac{\pi F\left(k_{1}\right)}{\left|f^{\prime}\left(k_{1}\right)\right|} .
\end{aligned}
$$

Equation 57 then becomes

$\pi \sin \sigma t \sum_{n=1}^{2} \frac{F_{n}\left(k_{n}\right)}{C_{g_{n}}\left(k_{n}\right)}$ 
in the limit, as $t \rightarrow \infty$. Here, $C_{g_{n}}(k)=\partial \omega_{n}(k) / \partial k$ is the group velocity for waves of the $n$-th mode. Finally, by using (61), the limit of $G^{(1)}$, as $t \rightarrow \infty$, is

$$
\begin{aligned}
G^{(1)}= & \cos \sigma t \sum_{n=-\infty}^{\infty}\left(\frac{1}{r_{n}}-\frac{1}{r_{1 n}}\right)+\cos \sigma t \int_{0}^{\infty} \frac{2 \cosh k h_{2} \sinh \left(k\left(z-h_{1}\right)\right) \sinh \left(k\left(\zeta-h_{1}\right)\right) J_{0}(k R)}{\sinh k h_{1}\left(\cosh k h_{1} \cosh k h_{2}+\gamma \sinh k h_{1} \sinh k h_{2}\right)} \mathrm{d} k \\
& +\cos \sigma t \sum_{n=1}^{2} \mathrm{pv} \int_{0}^{\infty} \frac{2 g k \omega_{n}^{2} P_{n}^{(1)}(k, z, \zeta) J_{0}(k R)}{\sinh k h_{1}\left(\cosh k h_{1} \cosh k h_{2}+\gamma \sinh k h_{1} \sinh k h_{2}\right)\left(\omega_{1}^{2}-\omega_{2}^{2}\right)\left(\omega_{n}^{2}-\sigma^{2}\right)} \mathrm{d} k \\
& +\sum_{n=1}^{2} \frac{\pi g k_{n} \sigma P_{n}^{(1)}\left(k_{n}, z, \zeta\right) J_{0}\left(k_{n} R\right) \sin \sigma t}{C_{g_{n}}\left(k_{n}\right) \sinh k_{n} h_{1}\left(\cosh k_{n} h_{1} \cosh k_{n} h_{2}+\gamma \sinh k_{n} h_{1} \sinh k_{n} h_{2}\right)\left(\omega_{1}^{2}\left(k_{n}\right)-\omega_{2}^{2}\left(k_{n}\right)\right)} .
\end{aligned}
$$

The above expression is the desired velocity potential in the upper fluid layer due to a stationary, oscillating source also in the upper layer. To obtain the potential of this source in the lower fluid layer, we apply the same procedures to $\Phi^{(2)}$ in (49) to yield:

$$
\begin{aligned}
G^{(2)}= & \cos \sigma t \int_{0}^{\infty} \frac{-2 \gamma \cosh \left(k\left(z+h_{2}\right)\right) \sinh \left(k\left(\zeta-h_{1}\right)\right) J_{0}(k R)}{\cosh k h_{1} \cosh k h_{2}+\gamma \sinh k h_{1} \sinh k h_{2}} \mathrm{~d} k \\
& +\cos \sigma t \sum_{n=1}^{2} \mathrm{pv} \int_{0}^{\infty} \frac{2 g k \omega_{n}^{2} P_{n}^{(2)}(k, z, \zeta) J_{0}(k R)}{\sinh k h_{1}\left(\cosh k h_{1} \cosh k h_{2}+\gamma \sinh k h_{1} \sinh k h_{2}\right)\left(\omega_{1}^{2}-\omega_{2}^{2}\right)\left(\omega_{n}^{2}-\sigma^{2}\right)} \mathrm{d} k \\
& +\sum_{n=1}^{2} \frac{\pi g k_{n} \sigma P_{n}^{(2)}\left(k_{n}, z, \zeta\right) J_{0}\left(k_{n} R\right) \sin \sigma t}{C_{g_{n}}\left(k_{n}\right) \sinh k_{n} h_{1}\left(\cosh k_{n} h_{1} \cosh k_{n} h_{2}+\gamma \sinh k_{n} h_{1} \sinh k_{n} h_{2}\right)\left(\omega_{1}^{2}\left(k_{n}\right)-\omega_{2}^{2}\left(k_{n}\right)\right)} .
\end{aligned}
$$

The dependence of $G^{(m)}$ on time $t$ can be separated from the other variables by expressing $G^{(m)}$ in terms of the complex potentials $g^{(m)}$ as follows:

$G^{(m)}(\xi, \eta, \zeta, x, y, z, t)=\mathfrak{R e}\left\{g^{(m)}(\xi, \eta, \zeta, x, y, z) \mathrm{e}^{-\mathrm{i} \sigma t}\right\}$,

where $\mathfrak{R e}$ denotes the real part of the expression, and $\mathrm{i}=\sqrt{-1}$. The complex potentials $g^{(m)}$ are then given by:

$$
\begin{aligned}
g^{(1)}= & \sum_{n=-\infty}^{\infty}\left(\frac{1}{r_{n}}-\frac{1}{r_{1 n}}\right)+\int_{0}^{\infty} \frac{2 \cosh k h_{2} \sinh \left(k\left(z-h_{1}\right)\right) \sinh \left(k\left(\zeta-h_{1}\right)\right) J_{0}(k R)}{\sinh k h_{1}\left(\cosh k h_{1} \cosh k h_{2}+\gamma \sinh k h_{1} \sinh k h_{2}\right)} \mathrm{d} k \\
& +\sum_{n=1}^{2} \mathrm{pv} \int_{0}^{\infty} \frac{2 g k \omega_{n}^{2} P_{n}^{(1)}(k, z, \zeta) J_{0}(k R)}{\sinh k h_{1}\left(\cosh k h_{1} \cosh k h_{2}+\gamma \sinh k h_{1} \sinh k h_{2}\right)\left(\omega_{1}^{2}-\omega_{2}^{2}\right)\left(\omega_{n}^{2}-\sigma^{2}\right)} \mathrm{d} k \\
& +\sum_{n=1}^{2} \frac{\mathrm{i} \pi g k_{n} \sigma P_{n}^{(1)}\left(k_{n}, z, \zeta\right) J_{0}\left(k_{n} R\right)}{C_{g_{n}}\left(k_{n}\right) \sinh k_{n} h_{1}\left(\cosh k_{n} h_{1} \cosh k_{n} h_{2}+\gamma \sinh k_{n} h_{1} \sinh k_{n} h_{2}\right)\left(\omega_{1}^{2}\left(k_{n}\right)-\omega_{2}^{2}\left(k_{n}\right)\right)}, \\
g^{(2)}= & \int_{0}^{\infty} \frac{-2 \gamma \cosh \left(k\left(z+h_{2}\right)\right) \sinh \left(k\left(\zeta-h_{1}\right)\right) J_{0}(k R)}{\cosh k h_{1} \cosh k h_{2}+\gamma \sinh k h_{1} \sinh k h_{2}} \mathrm{~d} k \\
& +\sum_{n=1}^{2} \mathrm{pv} \int_{0}^{\infty} \frac{2 g k \omega_{n}^{2} P_{n}^{(2)}(k, z, \zeta) J_{0}(k R)}{\sinh k h_{1}\left(\cosh k h_{1} \cosh k h_{2}+\gamma \sinh k h_{1} \sinh k h_{2}\right)\left(\omega_{1}^{2}-\omega_{2}^{2}\right)\left(\omega_{n}^{2}-\sigma^{2}\right)} \mathrm{d} k \\
& +\sum_{n=1}^{2} \frac{\mathrm{i} \pi g k_{n} \sigma P_{n}^{(2)}\left(k_{n}, z, \zeta\right) J_{0}\left(k_{n} R\right)}{C_{g_{n}}\left(k_{n}\right) \sinh k_{n} h_{1}\left(\cosh k_{n} h_{1} \cosh k_{n} h_{2}+\gamma \sinh k_{n} h_{1} \sinh k_{n} h_{2}\right)\left(\omega_{1}^{2}\left(k_{n}\right)-\omega_{2}^{2}\left(k_{n}\right)\right)} .
\end{aligned}
$$




\section{Velocity potentials of a translating and oscillating source}

A source with oscillating strength moving at a constant speed is normally used in boundary-integral methods to study the motions of a translating body in waves. The body is moving at a mean forward speed $U$ and is experiencing small oscillatory motions about its mean position due to wave actions. For this problem, a rectangular coordinate system $\bar{O} \bar{x} \bar{y} \bar{z}$ moving with the ship at speed $U$ along the positive $\bar{x}$-axis is adopted. The $\bar{x} \bar{y}$-plane coincides with the undisturbed interface between the two fluids, and the $\bar{z}$-axis is positive upward. The location of the translating source $(\bar{\xi}, \bar{\eta}, \bar{\zeta})$ is fixed in this moving system where $\bar{\zeta}>0$ since the source is restricted to the upper layer. In this section, we derive the source potentials, or Green functions $G^{(m)}$, in the upper and lower fluid layer where $m=1$ and 2, respectively. Application of $G^{(m)}$ in a boundary-integral or panel method to compute ship motions is analogous to the single-fluid case and will be pursued in future study. The theoretical development is however given here.

The Green functions $G^{(m)}$ can be obtained either by solving a boundary-value problem similar to (1)-(7) or by specifying the appropriate source strength and position in the transient source potentials and taking the limit as $t \rightarrow \infty$. As in the stationary case, the latter approach is more convenient and is the one adopted here. This approach is illustrated below only for the simpler function $G^{(2)}$ since the steps are essentially the same for both potentials. For $G^{(2)}$, we start with the transient source potential $\Phi^{(2)}$ in (49). The coordinate system $O x y z$ in this equation is stationary and is related to the moving system $\bar{O} \bar{x} \bar{y} \bar{z}$ through the equations: $x=\bar{x}+U t, y=\bar{y}$, and $z=\bar{z}$. Setting the source strength $v(t)=\cos \sigma t$ in (49) and expressing the source and field-point location in the moving frame, we can show that the first term of $G^{(2)}$ is simply given by:

$\cos \sigma t \int_{0}^{\infty} \frac{-2 \gamma \cosh \left(k\left(\bar{z}+h_{2}\right)\right) \sinh \left(k\left(\bar{\zeta}-h_{1}\right)\right) J_{0}(k \bar{R})}{\cosh k h_{1} \cosh k h_{2}+\gamma \sinh k h_{1} \sinh k h_{2}} \mathrm{~d} k$,

having taken the limit of $t \rightarrow \infty$. Here, $\bar{R}^{2}=(\bar{x}-\bar{\xi})^{2}+(\bar{y}-\bar{\eta})^{2}$. The remaining term in (49) contains contributions from both wave modes, and its limit requires further consideration. To simplify the notations, let us denote this term $G_{\text {wave }}^{(2)}$ and introduce the variables $D=\cosh k h_{1} \cosh k h_{2}+\gamma \sinh k h_{1} \sinh k h_{2}, \bar{X}=\bar{x}-\bar{\xi}, \bar{Y}=\bar{y}-\bar{\eta}$, and $\bar{\omega}=\bar{X} \cos \theta+\bar{Y} \sin \theta$. Using Eq. 42, we can write the Bessel function $J_{0}(k R(\tau))$ as:

$J_{0}(k R(\tau))=\frac{1}{2 \pi} \int_{-\pi}^{\pi} \mathrm{e}^{\mathrm{i} k(\bar{X} \cos \theta+\bar{Y} \sin \theta)} \mathrm{e}^{\mathrm{i} k U(t-\tau) \cos \theta} \mathrm{d} \theta=\frac{1}{2 \pi} \int_{-\pi}^{\pi} \mathrm{e}^{\mathrm{i} k \bar{\omega}} \mathrm{e}^{\mathrm{i} k U(t-\tau) \cos \theta} \mathrm{d} \theta$.

The term $G_{\text {wave }}^{(2)}$ then becomes:

$G_{\text {wave }}^{(2)}=\lim _{t \rightarrow \infty} \sum_{n=1}^{2} \int_{-\pi}^{\pi} \int_{0}^{\infty} \frac{g k \omega_{n} P_{n}^{(2)} \mathrm{e}^{\mathrm{i} k \bar{\omega}}}{\pi D \sinh k h_{1}\left(\omega_{1}^{2}-\omega_{2}^{2}\right)} \int_{0}^{t} \cos (\sigma \tau) \sin \left(\omega_{n}(t-\tau)\right) \mathrm{e}^{\mathrm{i} k U(t-\tau) \cos \theta} \mathrm{d} \tau \mathrm{d} k \mathrm{~d} \theta$.

The range of the $\theta$-integration can be reduced by making simple variable substitutions for $\theta$ in the quadrants $[-\pi,-\pi / 2]$ and $[\pi / 2, \pi]$ :

$G_{\text {wave }}^{(2)}=\lim _{t \rightarrow \infty} \sum_{n=1}^{2} \int_{-\frac{\pi}{2}}^{\frac{\pi}{2}} \int_{0}^{\infty} \frac{2 g k \omega_{n} P_{n}^{(2)}}{\pi D \sinh k h_{1}\left(\omega_{1}^{2}-\omega_{2}^{2}\right)} \int_{0}^{t} \cos (\sigma(t-\tau)) \sin \left(\omega_{n} \tau\right) \cos (k(\bar{\omega}+U \tau \cos \theta)) \mathrm{d} \tau \mathrm{d} k \mathrm{~d} \theta$.

To perform the $\tau$-integration, we first use the angle-sum identity for cosine to move the term involving $\bar{Y}$ in $\bar{\omega}$ outside the integral:

$G_{\text {wave }}^{(2)}=\lim _{t \rightarrow \infty}\left\{\sum_{n=1}^{2} \frac{-1}{\pi} \int_{0}^{\frac{\pi}{2}} \int_{0}^{\infty} \frac{g k \omega_{n} P_{n}^{(2)} \cos (k \bar{Y} \sin \theta)}{D \sinh k h_{1}\left(\omega_{1}^{2}-\omega_{2}^{2}\right)} \cdot T(t) \mathrm{d} k \mathrm{~d} \theta\right\}$, 
where

$T(t)=-4 \int_{0}^{t} \cos (\sigma(t-\tau)) \sin \left(\omega_{n} \tau\right) \cos (k(\bar{X}+U \tau) \cos \theta) \mathrm{d} \tau$.

Using the function-product relations for sine and cosine, we can express $T(t)$ in a more integratable form:

$$
\begin{aligned}
T(t)= & -\int_{0}^{t}\left\{\sin \left(D_{0} \tau+k \bar{X} \cos \theta-\sigma t\right)-\sin \left(D_{1,2} \tau+k \bar{X} \cos \theta-\sigma t\right)\right. \\
& \left.+\sin \left(D_{3} \tau+k \bar{X} \cos \theta+\sigma t\right)-\sin \left(D_{4} \tau+k \bar{X} \cos \theta+\sigma t\right)\right\} \mathrm{d} \tau,
\end{aligned}
$$

where $D_{0}=k U \cos \theta+\sigma+\omega_{n}, D_{1,2}=k U \cos \theta+\sigma-\omega_{n}, D_{3}=k U \cos \theta-\sigma+\omega_{n}$, and $D_{4}=k U \cos \theta-\sigma-\omega_{n}$. Performing the integration and simplifying the result, we have:

$$
\begin{aligned}
& T=\cos (k \bar{X} \cos \theta-\sigma t)\left\{\frac{\cos \left(D_{0} t\right)}{D_{0}}-\frac{\cos \left(D_{1,2} t\right)}{D_{1,2}}+\frac{2 \omega_{n}}{D_{0} D_{1,2}}\right\}-\sin (k \bar{X} \cos \theta-\sigma t)\left\{\frac{\sin \left(D_{0} t\right)}{D_{0}}-\frac{\sin \left(D_{1,2} t\right)}{D_{1,2}}\right\} \\
& +\cos (k \bar{X} \cos \theta+\sigma t)\left\{\frac{\cos \left(D_{3} t\right)}{D_{3}}-\frac{\cos \left(D_{4} t\right)}{D_{4}}+\frac{2 \omega_{n}}{D_{3} D_{4}}\right\}-\sin (k \bar{X} \cos \theta+\sigma t)\left\{\frac{\sin \left(D_{3} t\right)}{D_{3}}-\frac{\sin \left(D_{4} t\right)}{D_{4}}\right\} . \text { (74) }
\end{aligned}
$$

Next, we take the limit of $G_{\text {wave }}^{(2)}$ as $t \rightarrow \infty$ using (59) and (60). Application of these equations requires consideration of the poles of $G_{\text {wave }}^{(2)}$, or more specifically, the zeroes of the terms $D_{0}, D_{1,2}, D_{3}$ and $D_{4}$ for values of $\theta$ in the integration range $[0, \pi / 2]$. The term $D_{0}$ has no zeroes in this range, but $D_{3}$ and $D_{4}$ each has one zero at $k=k_{3, n}$ and $k=k_{4, n}$, respectively. They are given implicitly by the following equations:

$k_{3, n} U \cos \theta-\sigma+\omega_{n}\left(k_{3, n}\right)=0$ and $k_{4, n} U \cos \theta-\sigma-\omega_{n}\left(k_{4, n}\right)=0$.

The zero $k_{4, n}$ is always greater than $k_{3, n}$, and both zeroes exist for any $\theta \in[0, \pi / 2]$. For $D_{1,2}$, there are 2 roots to the equation $k U \cos \theta+\sigma-\omega_{n}=0$ for certain values of $\theta$. We shall denote these roots as $k_{1, n}$ and $k_{2, n}$ with $k_{1, n}<k_{2, n}$ and determine $\theta$ where they exist. For each frequency $\sigma$, we can define a wavenumber $k_{n}^{*}$ satisfying the equation:

$\sigma=\omega_{n}\left(k_{n}^{*}\right)-k_{n}^{*} C_{g_{n}}\left(k_{n}^{*}\right)$,

where $C_{g_{n}}=\partial \omega_{n}(k) / \partial k$ is the group velocity for wave mode $n$. Let $U_{n}^{*}=C_{g_{n}}\left(k_{n}^{*}\right)$ be the group velocity corresponding to $k_{n}^{*}$. For $U<U_{n}^{*}$, both $k_{1, n}$ and $k_{2, n}$ exist for $\theta \in[0, \pi / 2]$. However, when $U>U_{n}^{*}, k_{1, n}$ and $k_{2, n}$ exist only for $\theta>\theta_{n}^{*}=\cos ^{-1}\left(U_{n}^{*} / U\right)$.

With the poles of $G_{\text {wave }}^{(2)}$ defined, we can now perform the limit as $t \rightarrow \infty$ in (71) after substituting for $T(t)$ in (74). Using the formulas in (59) and (60), we obtain:

$$
\begin{aligned}
G_{\text {wave }}^{(2)}= & \sum_{n=1}^{\infty}\left\{\frac{-2}{\pi} \int_{0}^{\frac{\pi}{2}} \mathrm{pv} \int_{0}^{\infty} \omega_{n} H_{n}^{(2)}\left[\frac{\cos (k \bar{X} \cos \theta+\sigma t)}{(k U \cos \theta-\sigma)^{2}-\omega_{n}^{2}}+\frac{\cos (k \bar{X} \cos \theta-\sigma t)}{(k U \cos \theta+\sigma)^{2}-\omega_{n}^{2}}\right] \mathrm{d} k \mathrm{~d} \theta\right. \\
& +\int_{\theta_{n}^{*}}^{\frac{\pi}{2}} \frac{H_{n}^{(2)}\left(k_{1, n}\right) \sin \left(k_{1, n} \bar{X} \cos \theta-\sigma t\right)}{U \cos \theta-C_{g_{n}}\left(k_{1, n}\right)}-\int_{\theta_{n}^{*}}^{\frac{\pi}{2}} \frac{H_{n}^{(2)}\left(k_{2, n}\right) \sin \left(k_{2, n} \bar{X} \cos \theta-\sigma t\right)}{U \cos \theta-C_{g_{n}}\left(k_{2, n}\right)} \\
& \left.+\int_{0}^{\frac{\pi}{2}} \frac{H_{n}^{(2)}\left(k_{3, n}\right) \sin \left(k_{3, n} \bar{X} \cos \theta+\sigma t\right)}{U \cos \theta+C_{g_{n}}\left(k_{3, n}\right)}-\int_{0}^{\frac{\pi}{2}} \frac{H_{n}^{(2)}\left(k_{4, n}\right) \sin \left(k_{4, n} \bar{X} \cos \theta+\sigma t\right)}{U \cos \theta-C_{g_{n}}\left(k_{4, n}\right)}\right\},
\end{aligned}
$$

where

$$
H_{n}^{(2)}=\frac{g k \omega_{n} P_{n}^{(2)} \cos (k \bar{Y} \sin \theta)}{D \sinh k h_{1}\left(\omega_{1}^{2}-\omega_{2}^{2}\right)} \quad \text { and } \quad \theta_{n}^{*}= \begin{cases}0 & \text { if } U<U_{n}^{*} \\ \cos ^{-1}\left(U_{n}^{*} / U\right) & \text { if } U>U_{n}^{*}\end{cases}
$$




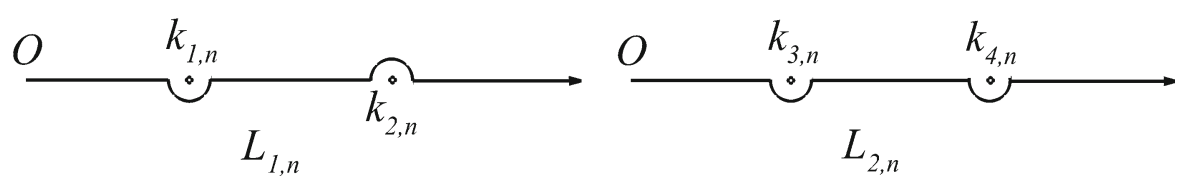

Fig. 2 Paths of integration $L_{1, n}$ and $L_{2, n}$

The complete Green function $G^{(2)}$ in the lower fluid can be obtained by summing (67) and (77). For the Green function $G^{(1)}$ in the upper fluid, we apply the same procedures to the transient source $\Phi^{(1)}$ in (51) to yield:

$G^{(1)}=\cos \sigma t \sum_{n=-\infty}^{\infty}\left(\frac{1}{\bar{r}_{n}}-\frac{1}{\bar{r}_{1 n}}\right)+\cos \sigma t \int_{0}^{\infty} \frac{2 \cosh k h_{2} \sinh \left(k\left(\bar{z}-h_{1}\right)\right) \sinh \left(k\left(\bar{\zeta}-h_{1}\right)\right) J_{0}(k \bar{R})}{\sinh k h_{1}\left(\cosh k h_{1} \cosh k h_{2}+\gamma \sinh k h_{1} \sinh k h_{2}\right)} \mathrm{d} k+G_{\text {wave }}^{(1)}$,

where $G_{\text {wave }}^{(1)}$ is exactly the same as $G_{\text {wave }}^{(2)}$ in (77) and (78) except $H_{n}^{(2)}$ and $P_{n}^{(2)}$ are replaced by $H_{n}^{(1)}$ and $P_{n}^{(1)}$, respectively.

In typical numerical calculations, the complex forms of the Green functions $g^{(m)}$ are normally used. They are related to $G^{(m)}$ through (64). The functions $g^{(m)}$ can be written in a more compact form using path integrals as follows:

$$
\begin{aligned}
g^{(1)}= & \sum_{n=-\infty}^{\infty}\left(\frac{1}{\bar{r}_{n}}-\frac{1}{\bar{r}_{1 n}}\right)+\int_{0}^{\infty} \frac{2 \cosh k h_{2} \sinh \left(k\left(\bar{z}-h_{1}\right)\right) \sinh \left(k\left(\bar{\zeta}-h_{1}\right)\right) J_{0}(k \bar{R})}{\sinh k h_{1}\left(\cosh k h_{1} \cosh k h_{2}+\gamma \sinh k h_{1} \sinh k h_{2}\right)} \mathrm{d} k \\
& +\sum_{n=1}^{2}\left\{\frac{2}{\pi} \int_{0}^{\theta_{n}^{*}} \int_{0}^{\infty} F_{n}^{(1)}(k, \theta) \mathrm{d} k \mathrm{~d} \theta+\frac{2}{\pi} \int_{\theta_{n}^{*}}^{\frac{\pi}{2}} \int_{L_{1, n}} F_{n}^{(1)}(k, \theta) \mathrm{d} k \mathrm{~d} \theta+\frac{2}{\pi} \int_{\frac{\pi}{2}}^{\pi} \int_{L_{2, n}} F_{n}^{(1)}(k, \theta) \mathrm{d} k \mathrm{~d} \theta\right\}, \\
g^{(2)}= & -\int_{0}^{\infty} \frac{2 \gamma \cosh \left(k\left(\bar{z}+h_{2}\right)\right) \sinh \left(k\left(\bar{\zeta}-h_{1}\right)\right) J_{0}(k \bar{R})}{\cosh k h_{1} \cosh k h_{2}+\gamma \sinh k h_{1} \sinh k h_{2}} \mathrm{~d} k \\
& +\sum_{n=1}^{2}\left\{\frac{2}{\pi} \int_{0}^{\theta_{n}^{*}} \int_{0}^{\infty} F_{n}^{(2)}(k, \theta) \mathrm{d} k \mathrm{~d} \theta+\frac{2}{\pi} \int_{\theta_{n}^{*}}^{\frac{\pi}{2}} \int_{L_{1, n}} F_{n}^{(2)}(k, \theta) \mathrm{d} k \mathrm{~d} \theta+\frac{2}{\pi} \int_{\frac{\pi}{2}}^{\pi} \int_{L_{2, n}} F_{n}^{(2)}(k, \theta) \mathrm{d} k \mathrm{~d} \theta\right\},
\end{aligned}
$$

where

$F_{n}^{(m)}(k, \theta)=\frac{g k \omega_{n}^{2} P_{n}^{(m)} \mathrm{e}^{-\mathrm{i} k(\bar{x}-\bar{\xi}) \cos \theta} \cos (k(\bar{y}-\bar{\eta}) \sin \theta)}{\sinh k h_{1}\left(\cosh k h_{1} \cosh k h_{2}+\gamma \sinh k h_{1} \sinh k h_{2}\right)\left(\omega_{1}^{2}-\omega_{2}^{2}\right)\left(\omega_{n}^{2}-(k U \cos \theta+\sigma)^{2}\right)}$,

and the paths $L_{1, n}$ and $L_{2, n}$ are as shown in Fig. 2. The forms of $g^{(1)}$ and $g^{(2)}$ above are similar to the Green function for a single-layer fluid as given in Wehausen and Laitone [24, p. 493, Eq. 13.52]. Angle $\theta_{n}^{*}$ and paths $L_{1, n}, L_{2, n}$ are analogous to the angle $\gamma$ and the paths $L_{1}, L_{2}$ of the cited reference.

\section{Radiation and diffraction of waves in a two-layer fluid}

To illustrate how the velocity potentials derived in the previous sections can be used to solve practical ocean-engineering problems where density stratification exists, we present, in this section, the solution for the motions of a floating structure in waves in a two-layer fluid of finite depth. The solution is obtained using the boundary-integral method and the velocity potentials of a stationary, oscillating source derived in Sect. 3. To study the motions in 
waves, one must first investigate the types of ambient waves that can propagate in a two-layer fluid. This is done in Sect. 5.1 below. It is followed by the mathematical description of the problem and solution in integral form using Green's theorem in Sect. 5.2. Numerical evaluation of the source potentials and numerical solution of the integral equation are given in Sects. 5.3 and 5.4, respectively. Finally, numerical results for a floating barge are presented in Sect. 5.5 where the effects of internal waves on the barge motions are shown.

\subsection{Incident waves in a two-layer fluid of finite depth}

A long-crested incident-wave system in a two-layer fluid contains both the normal free-surface waves as well as internal waves on the interface between the two fluid layers. As will be shown below, there are two possible incident-wave systems that can exist at any frequency $\sigma$, each propagating at its own distinct wavelength. We refer to a system with surface waves that closely resemble waves in a single fluid as propagating in the "surface-wave mode". In this system, the displacements of the fluid particles decrease with depth, and the amplitudes of the internal waves are consequently smaller than those of the surface waves. However, when a wave system propagates in the "internal-wave mode", fluid displacement increases as the depth approaches the fluid interface. Regardless of the initial mode of propagation, once the incident-wave system impacts upon a floating structure, waves of both modes are scattered, and the exciting forces acting on the structure can differ significantly from the single-fluid case.

To calculate the motions of a structure due to these incident-wave systems, we start with the derivation of the velocity potentials of these waves. Figure 3 shows a system of plane progressive waves propagating at an angle $\beta$ with respect to the positive $x$-axis. Let us denote the surface and interface elevations of this wave system by $\zeta^{(1)}$ and $\zeta^{(2)}$, respectively. We can then write $\zeta^{(m)}, m=1,2$ in complex notation as follows:

$\zeta^{(m)}(x, y, t)=\mathfrak{R e}\left\{a_{0}^{(m)} \mathrm{e}^{\mathrm{i}(k(x \cos \beta+y \sin \beta)-\sigma t)}\right\}$,

where $a_{0}^{(1)}$ and $a_{0}^{(2)}$ are the amplitudes of the surface and internal waves, and $k, \sigma$ are the wavenumber and frequency, respectively. The velocity potentials $\Phi_{0}^{(m)}$ of these waves satisfy the usual Laplace equation and also the following kinematic and dynamic conditions on the free surface:

$\Phi_{0, z}^{(1)}-\zeta_{t}^{(1)}=0$ and $\Phi_{0, t}^{(1)}+g \zeta^{(1)}=0, \quad z=h_{1}$,

and on the interface

$\Phi_{0, z}^{(1)}=\Phi_{0, z}^{(2)}=\zeta_{t}^{(2)} \quad$ and $\gamma\left(\Phi_{0, t}^{(1)}+g \zeta^{(1)}\right)=\Phi_{0, t}^{(2)}+g \zeta^{(2)}, \quad z=0$.

Also, $\Phi_{0}^{(2)}$ must satisfy the no-flux, bottom condition

$\Phi_{0, z}^{(2)}=0, \quad z=-h_{2}$.

To solve for the potentials $\Phi_{0}^{(m)}$, we assume the following forms:

$\Phi_{0}^{(1)}=\mathfrak{R e}\left\{\left(A_{0} \cosh k z+B_{0} \sinh k z\right) \mathrm{e}^{\mathrm{i}(k(x \cos \beta+y \sin \beta)-\sigma t)}\right\}$,

$\Phi_{0}^{(2)}=\mathfrak{R e}\left\{C_{0} \cosh \left(k\left(z+h_{2}\right)\right) \mathrm{e}^{\mathrm{i}(k(x \cos \beta+y \sin \beta)-\sigma t)}\right\}$.

Clearly, $\Phi_{0}^{(m)}$ satisfies the Laplace equation and the bottom condition in (86). Substituting $\zeta^{(m)}$ in (83) and $\Phi_{0}^{(m)}$ in (87) and (88) in the remaining boundary conditions in (84) and (85), we obtain the following expressions:

$A_{0}=\mathrm{i} a_{0}^{(1)} \cosh k h_{1}\left(\sigma^{2} \tanh k h_{1}-g k\right) /(\sigma k)$,

$B_{0}=-\mathrm{i} a_{0}^{(1)} \cosh k h_{1}\left(\sigma^{2}-g k \tanh k h_{1}\right) /(\sigma k)$,

$C_{0}=B_{0} / \sinh k h_{2}$,

$a_{0}^{(2)}=a_{0}^{(1)} \cosh k h_{1}\left(\sigma^{2}-g k \tanh k h_{1}\right) / \sigma^{2}$,

$\sigma^{4}-\frac{g k\left(t_{1}+t_{2}\right)}{1+\gamma t_{1} t_{2}} \sigma^{2}+\frac{\varepsilon(g k)^{2} t_{1} t_{2}}{1+\gamma t_{1} t_{2}}=0$, 


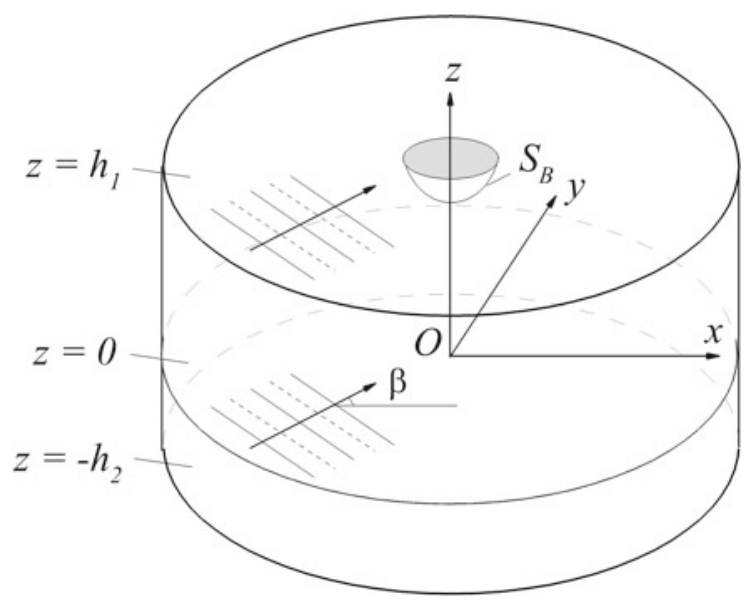

Fig. 3 Floating structure in incident-wave systems

where $t_{1}=\tanh k h_{1}$ and $t_{2}=\tanh k h_{2}$ as defined in Sect 2.2. Equation 92 indicates that $a_{0}^{(2)}$ is dependent on $a_{0}^{(1)}$. Thus, to describe an incident-wave system, one only needs to specify the amplitude of either the surface waves or the internal waves and not both. Equation 93 relates the wavenumber $k$ to the wave frequency $\sigma$ and can be solved to yield the dispersion relations. This quartic equation has only two real roots that are physically possible. They are given by

$\sigma=\omega_{1}(k)=\left\{\frac{g k\left[\left(t_{1}+t_{2}\right)+\sqrt{\left(t_{1}+t_{2}\right)^{2}-4 \varepsilon t_{1} t_{2}\left(1+\gamma t_{1} t_{2}\right)}\right]}{2\left(1+\gamma t_{1} t_{2}\right)}\right\}^{1 / 2}$,
$\sigma=\omega_{2}(k)=\left\{\frac{g k\left[\left(t_{1}+t_{2}\right)-\sqrt{\left(t_{1}+t_{2}\right)^{2}-4 \varepsilon t_{1} t_{2}\left(1+\gamma t_{1} t_{2}\right)}\right]}{2\left(1+\gamma t_{1} t_{2}\right)}\right\}^{1 / 2}$.

Equations 94 and 95 have two solutions $k_{1}$ and $k_{2}$, respectively, and they express the two possible dispersion relations for waves propagating in a two-layer fluid of finite depth. If the two fluid layers have the same density, then $\gamma=1, \varepsilon=0$, and (94) and (95) reduce to:

$\sigma=\omega_{1}(k)=\sqrt{g k \tanh \left(k\left(h_{1}+h_{2}\right)\right)}$ and $\sigma=\omega_{2}(k)=0$.

The first expression is the same as the dispersion relation for surface waves in a single fluid of depth $h_{1}+h_{2}$. Thus, we denote the wave mode associated with the dispersion relation in (94) as the "surface-wave mode", and the mode associated with (95) as the "internal-wave mode".

In summary, for a given frequency $\sigma$, there are two possible incident-wave systems corresponding to the surface-wave mode with wavenumber $k_{1}$ and to the internal-wave mode with wavenumber $k_{2}$. Each system contains both surface and internal waves. When $k_{1}$ is substituted in (92), we can show that the amplitude ratio $a_{0}^{(1)} / a_{0}^{(2)}>1$. Thus, for the surface-wave mode, both the surface and internal waves are in phase, and the amplitude of the surface waves is greater than the internal waves. When we insert $k_{2}$ into (92), we have $-1<a_{0}^{(1)} / a_{0}^{(2)}<0$. Thus, for the internal-wave mode, the surface and internal waves are $180^{\circ}$ out of phase, and the amplitude of the internal waves is greater than that of the surface waves. The velocity potentials $\Phi_{0}^{(m)}$ of the incident-wave system are obtained by 
substituting $A_{0}, B_{0}, C_{0}$ in (89)-(91) in (87) and (88):

$$
\begin{aligned}
& \Phi_{0}^{(1)}=\mathfrak{R e}\{ \frac{\mathrm{i} a_{0}^{(1)} \cosh k_{n} h_{1}}{\sigma k_{n}}\left(\left(\sigma^{2} \tanh k_{n} h_{1}-g k_{n}\right) \cosh k_{n} z\right. \\
&\left.\left.-\left(\sigma^{2}-g k_{n} \tanh k_{n} h_{1}\right) \sinh k_{n} z\right) \mathrm{e}^{\mathrm{i}\left(k_{n}(x \cos \beta+y \sin \beta)-\sigma t\right)}\right\}, \\
& \Phi_{0}^{(2)}=\mathfrak{R e}\left\{-\frac{\mathrm{i} a_{0}^{(1)} \cosh k_{n} h_{1}}{\sigma k_{n} \sinh k_{n} h_{2}}\left(\sigma^{2}-g k_{n} \tanh k_{n} h_{1}\right) \cosh \left(k_{n}\left(z+h_{2}\right)\right) \mathrm{e}^{\mathrm{i}\left(k_{n}(x \cos \beta+y \sin \beta)-\sigma t\right)}\right\} .
\end{aligned}
$$

Alternatively, we can also express $\Phi_{0}^{(m)}$ in terms of the amplitude of the internal waves $a_{0}^{(2)}$ using the amplitude ratio in (92):

$$
\begin{aligned}
& \Phi_{0}^{(1)}=\mathfrak{R e}\left\{\frac{\mathrm{i} \sigma a_{0}^{(2)}}{k_{n}}\left(\frac{\sigma^{2} \tanh k_{n} h_{1}-g k_{n}}{\sigma^{2}-g k_{n} \tanh k_{n} h_{1}} \cosh k_{n} z-\sinh k_{n} z\right) \mathrm{e}^{\mathrm{i}\left(k_{n}(x \cos \beta+y \sin \beta)-\sigma t\right)}\right\}, \\
& \Phi_{0}^{(2)}=\mathfrak{R e}\left\{-\frac{\mathrm{i} \sigma a_{0}^{(2)}}{k_{n} \sinh k_{n} h_{2}} \cosh \left(k_{n}\left(z+h_{2}\right)\right) \mathrm{e}^{\mathrm{i}\left(k_{n}(x \cos \beta+y \sin \beta)-\sigma t\right)}\right\} .
\end{aligned}
$$

\subsection{Decomposition of potentials}

Consider a rigid body oscillating in the upper fluid layer under the action of an incident-wave system of frequency $\sigma$ (see Fig. 3). This wave system can be of either wave mode, $n=1$ or 2 , and its velocity potentials $\Phi_{0}^{(m)}$ are given in (97) and (98). Let us denote the translational displacements of the body in the $x-, y$-, and $z$-direction as $\zeta_{1}(t), \zeta_{2}(t)$, and $\zeta_{3}(t)$, respectively, and the angular displacement components as $\zeta_{4}(t), \zeta_{5}(t)$, and $\zeta_{6}(t)$.

The total velocity potentials $\Phi^{(1)}$ and $\Phi^{(2)}$ in the upper and lower fluid layers must satisfy the Laplace equation as well as the boundary conditions in (3)-(6). In addition, since the body is floating in the upper fluid layer, $\Phi^{(1)}$ must also satisfy the following linearized kinematic condition on the surface of the body in its resting position (see [25]):

$\Phi_{, n}^{(1)}=\vec{V} \cdot \vec{n}$,

where $\vec{V}$ is the velocity of a point on the body surface, and $\vec{n}$ is the unit normal vector pointing into the body. Since the governing equations and boundary conditions are linear, the potential $\Phi^{(m)}(x, y, z, t)$ can be decomposed into the following components:

$\Phi^{(m)}=\Phi_{0}^{(m)}+\Phi_{7}^{(m)}+\sum_{j=1}^{6} \Phi_{j}^{(m)}$,

where $\Phi_{0}^{(m)}$ is the incident-wave potential, $\Phi_{7}^{(m)}$ is the diffraction potential, and $\Phi_{j}^{(m)}, j=1,2, \ldots, 6$ are the radiation potentials associated with the six degrees of freedom of body motions in otherwise calm water. Note that the mode of the incident wave determines $\Phi_{0}^{(m)}$, but it does not affect the formulation of the problem otherwise. For time-harmonic motions, we can express $\zeta_{j}(t)$ as:

$\zeta_{j}(t)=\mathfrak{R e}\left\{a_{j} \mathrm{e}^{-\mathrm{i} \sigma t}\right\}, \quad j=1,2, \ldots, 6$,

where $a_{j}$ are the complex amplitudes of the displacements. The potentials $\Phi^{(m)}$ can also be expressed in the same manner:

$\Phi^{(m)}(x, y, z, t)=\mathfrak{R e}\left\{\phi^{(m)}(x, y, z) \mathrm{e}^{-\mathrm{i} \sigma t}\right\}$. 
Applying the decomposition in (102), we can write $\phi^{(m)}$ as

$\phi^{(m)}(x, y, z)=\sum_{j=0}^{7}-\mathrm{i} \sigma a_{j} \phi_{j}^{(m)}(x, y, z)$,

where $a_{0}=a_{7}$ is the amplitude of the incident wave. If the incident wave is of the surface-wave mode, then $a_{0}=a_{0}^{(1)}$, and if it is of the internal-wave mode, then $a_{0}=a_{0}^{(2)}$. The potential $\phi_{0}^{(m)}$ can be obtained from (97)-(98). The other potentials $\phi_{j}^{(m)}, j=1, \ldots, 7$ satisfy the Laplace equation, and from (3)-(6), their boundary conditions are:

$\phi_{j, z}^{(1)}-\frac{\sigma^{2}}{g} \phi_{j}^{(1)}=0, \quad z=h_{1}$,

$\phi_{j, z}^{(1)}=\phi_{j, z}^{(2)}, \quad z=0$,

$\gamma\left(\phi_{j, z}^{(1)}-\frac{\sigma^{2}}{g} \phi_{j}^{(1)}\right)=\phi_{j, z}^{(2)}-\frac{\sigma^{2}}{g} \phi_{j}^{(2)}, \quad z=0$,

$\phi_{j, z}^{(2)}=0, \quad z=-h_{2}$,

From (101), we have

$\phi_{j, n}^{(1)}=n_{j}, \quad j=1,2, \ldots, 6$,

$\phi_{7, n}^{(1)}=-\phi_{0, n}^{(1)}=n_{7}$,

where $n_{1}, n_{2}, n_{3}$ are the $x$-, $y$-, $z$-components of $\vec{n}$, and $n_{4}, n_{5}, n_{6}$ are the $x$-, $y$-, z-components of $\left(\vec{r}-\vec{r}_{G}\right) \times \vec{n}$. Here $\vec{r}$ is the position vector of a point on the body surface, and $\vec{r}_{G}$ is the position vector of the body's center of gravity. Finally, to make the problem unique, we need to impose the appropriate radiation condition which requires the radiated and diffracted waves are outgoing and have the proper amplitude behavior at infinity.

To solve for $\phi_{j}^{(m)}$, we use Green's theorem and the Green functions $g^{(m)}$ for a stationary, oscillating source in (65) and (66). The potential $\phi_{j}^{(m)}$ can be expressed in terms of $g^{(m)}$ as:

$\phi_{j}^{(m)}=\int_{S_{B}} v_{j}(\xi, \eta, \zeta) g^{(m)}(\xi, \eta, \zeta, x, y, z) \mathrm{d} S$,

where $S_{B}$ is the surface of the body in its resting position, and $v_{j}$ is the source strength distribution on $S_{B}$ for the $j$ th motion. Substituting (112) in (110) and (111) yields the following Fredholm integral equation of the second kind:

$2 \pi v_{j}+\int_{S_{B}} v_{j} \frac{\partial g^{(1)}}{\partial n} \mathrm{~d} S=n_{j}, \quad j=1,2, \ldots, 7$.

This equation can be solved for the source strength $v_{j}$ which is then used in (112) to compute the potential $\phi_{j}^{(m)}$. The hydrodynamic force and moment acting on the body can be written as

$\vec{F}=\int_{S_{B}} p \vec{n} \mathrm{~d} S$ and $\vec{M}=\int_{S_{B}} p\left(\vec{r}-\vec{r}_{G}\right) \times \vec{n} \mathrm{~d} S$,

where $p$ is the fluid pressure which can be obtained from Euler's integral as

$p=\mathfrak{R e}\left\{\sum_{j=0}^{7} \rho_{1} \sigma^{2} a_{j} \phi_{j}^{(1)} \mathrm{e}^{-\mathrm{i} \sigma t}\right\}$.

If we denote the $x$-, $y$-, $z$-components of $\vec{F}$ as $F_{1}, F_{2}, F_{3}$, and the $x$-, $y$-, $z$-components of $\vec{M}$ as $F_{4}, F_{5}, F_{6}$, and $F_{i}=\mathfrak{R e}\left\{f_{i} \mathrm{e}^{-\mathrm{i} \sigma t}\right\}$, then using Eqs. 114 and 115, we can write

$f_{i}=\sum_{j=0}^{7} \rho_{1} \sigma^{2} a_{j} \int_{S_{B}} \phi_{j}^{(1)} n_{i} \mathrm{~d} S$, 
for $i=1,2, \ldots, 6$. Following the usual convention, we now define the wave-exciting force per unit wave amplitude $X_{i}$ as

$X_{i}=\rho_{1} \sigma^{2} \int_{S_{B}}\left(\phi_{0}^{(1)}+\phi_{7}^{(1)}\right) n_{i} \mathrm{~d} S$,

and the "added-masses" and "damping coefficients" as

$\mu_{i j}+\frac{i}{\sigma} \lambda_{i j}=\rho_{1} \int_{S_{B}} \phi_{j}^{(1)} n_{i} \mathrm{~d} S$.

In terms of these new variables, the hydrodynamic forces become

$f_{i}=a_{0} X_{i}+\sum_{j=1}^{6}\left(\sigma^{2} \mu_{i j} a_{j}+\mathrm{i} \sigma \lambda_{i j} a_{j}\right)$ for $i=1,2, \ldots, 6$.

The wave-exciting force depends on $\phi_{0}^{(1)}$ and, thus, on the mode of the incident wave. The added masses and damping coefficients, however, depend only on the body geometry and not the body motion or the incident-wave mode. Once $X_{i}, \mu_{i j}$, and $\lambda_{i j}$ are known, it is a simple matter to obtain the motion amplitudes $a_{j}$ using Newton's second law. While all this analysis is analogous to the single-fluid problem, the intricacies in the two-layer fluid problem lie in obtaining the Green function $g^{(1)}$ in (65) for a stationary, oscillating source.

\subsection{Numerical evaluation of $g^{(1)}$}

In this example, we are concerned with the motions of bodies floating in the upper fluid layer and not intersecting the fluid interface. Thus, we only need to evaluate $g^{(1)}$ and not $g^{(2)}$. These two functions, however, have similar forms, and the same numerical method can be used for their evaluation.

The first term of $g^{(1)}$, Eq. 65, represents an infinite array of Rankine sources and sinks. It is evaluated using the method presented in [26, pp. 112-113] which employs Fourier series and Chebyshev expansion. The integrals of $g^{(1)}$ are computed using an adaptive quadrature with Simpson's rule; see [27, pp. 328-332]. For the principal-valued integrals, the poles $k_{n}$ are obtained using Newton's method, and these singularities are removed using the procedure in [28] before the numerical integration is performed.

\subsection{Integral-equation solution}

Numerical results are obtained for a floating barge, modeled here as a rectangular box. A brief review of integralequation formulations may be found in e.g. [29]. Here, the low-order panel method of Hess and Smith [30] is used for simplicity. Higher-order panel methods such as the one proposed by Hamilton and Yeung [31] can be easily adopted.

To solve (113), we approximate the body surface by $N$ plane, quadrilateral panels. For the rectangular box in our example, this geometrical discretization is adequate and will not leave any gaps between adjacent panels. For each panel $p$, we assume the source strength $v_{j, p}$ to be constant and define the control point $\left(x_{p}, y_{p}, z_{p}\right)$ as the null point of the panel (see e.g. [30]). Application of (113) to each panel $p$ will yield the following system of linear equations

$$
\sum_{q=1}^{N} A_{p, q} v_{j, q}=n_{j, p}, \quad p=1,2, \ldots, N
$$

where $n_{j, p}$ is the component $n_{j}$ evaluated at the control point of panel $p$, and the elements of $A_{p, q}$ are defined as

$$
A_{p, q}= \begin{cases}2 \pi, & p=q, \\ \int_{S_{q}} \frac{\partial g^{(1)}}{\partial n}\left(\xi, \eta, \zeta, x_{p}, y_{p}, z_{p}\right) \mathrm{d} S, & p \neq q,\end{cases}
$$


where $S_{q}$ is the surface area of panel $q$. To perform the integration over $S_{q}$, we use Gaussian quadrature with 3 points in each direction for a total of 9 points for each panel. This system of equations is then solved using the direct method of Gaussian elimination. The potential $\phi_{j}^{(1)}$, which is also constant over each panel $p$, is evaluated at the control point as

$\phi_{j}^{(1)}\left(x_{p}, y_{p}, z_{p}\right)=\sum_{q=1}^{N} v_{j, q} \int_{S_{q}} g^{(1)}\left(\xi, \eta, \zeta, x_{p}, y_{p}, z_{p}\right) \mathrm{d} S$.

The wave-exciting forces and the added-mass and damping coefficients are computed in the same manner as well.

\subsection{Floating barge in a two-layer fluid of finite depth}

The wave-exciting forces and hydrodynamic coefficients are computed for a barge, modeled here as a rectangular box. The dimensions of the box are chosen so that comparison with the numerical results of Endo [28] for a single-layer fluid is possible. The box has length $L$ and beam $B$ each of $90 \mathrm{~m}$, and its draft $D$ is $40 \mathrm{~m}$. It floats on the free surface with no forward velocity. A total number of 192 panels is used to discretize the surface of the box. The results obtained agree with those using 432 panels, thus, verifying the convergence of the numerical solution.

To study the effects of density ratio $\gamma$ on the wave forces and motions of the box, we obtain solutions for $\gamma=1$, $0.9,0.7,0.1$, recalling that $\gamma=1-\varepsilon$. For these runs, the depths of the fluid layers are fixed, $h_{1}=1.2 D$ and $h_{2}=0.4 D$. When $\gamma=1$, the two-layer fluid becomes a single-layer fluid of depth $h=h_{1}+h_{2}=1.6 D$, and the results can be compared with those of Endo [28] for the same depth. As $\gamma$ approaches zero, i.e., $\rho_{2}$ becomes infinitely large, the lower fluid behaves more like a rigid block, and we would expect the results to approach those for a single-layer fluid of depth $h_{1}$, the depth of the upper fluid layer. To illustrate this behavior, we include the results for a single-layer fluid of depth $h_{1}$ in the plots as well.

Figures 4, 5, 6, 7, 8 and 9 show the added-masses and damping coefficients for surge, heave, and pitch. In these figures, $\nabla$ denotes the volume of the box displacement. Results for $\gamma=1$ and $h=1.6 D$ agree quite well with those of Endo [28]. Also, the results for $\gamma=0.1$ approximate those for a single-layer-fluid of depth $h=1.2 D$ as expected. The results for $\gamma=0.7,0.9$ are in between these two limits, and when compared to the single-layer-fluid case, they show the effects of internal waves on the hydrodynamic coefficients of the box. At high frequencies, these results are similar to those for a single-layer fluid, indicating internal waves are less important. However, at

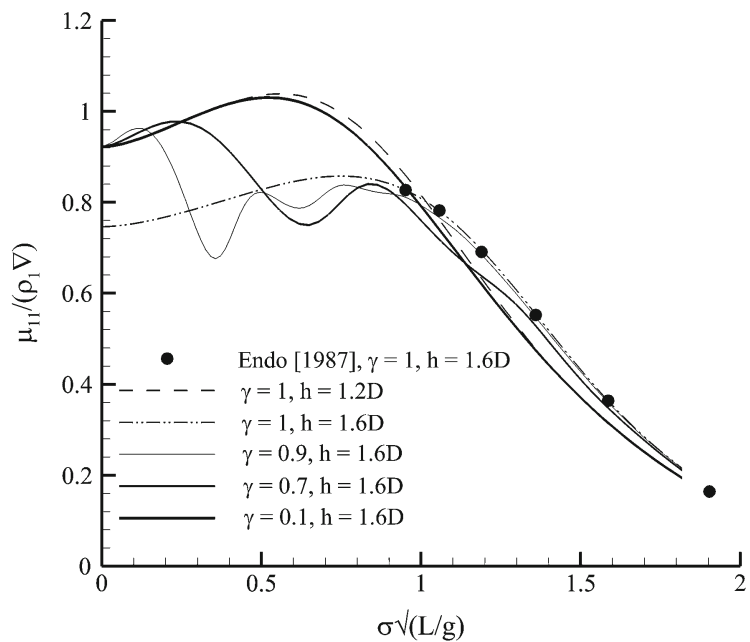

Fig. 4 Surge added mass of a rectangular box, $h_{1}=1.2 D$, $h_{2}=0.4 D$

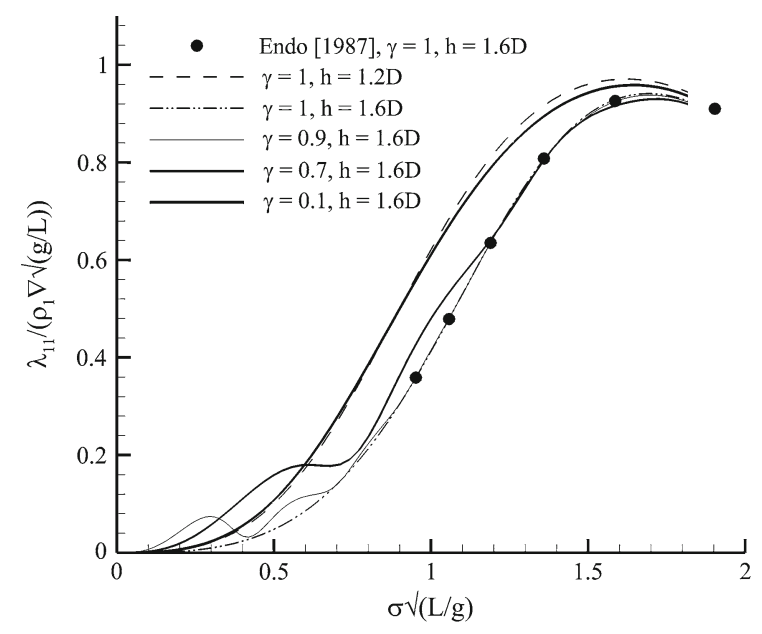

Fig. 5 Surge damping coefficient of a rectangular box, $h_{1}=1.2 D, h_{2}=0.4 D$ 


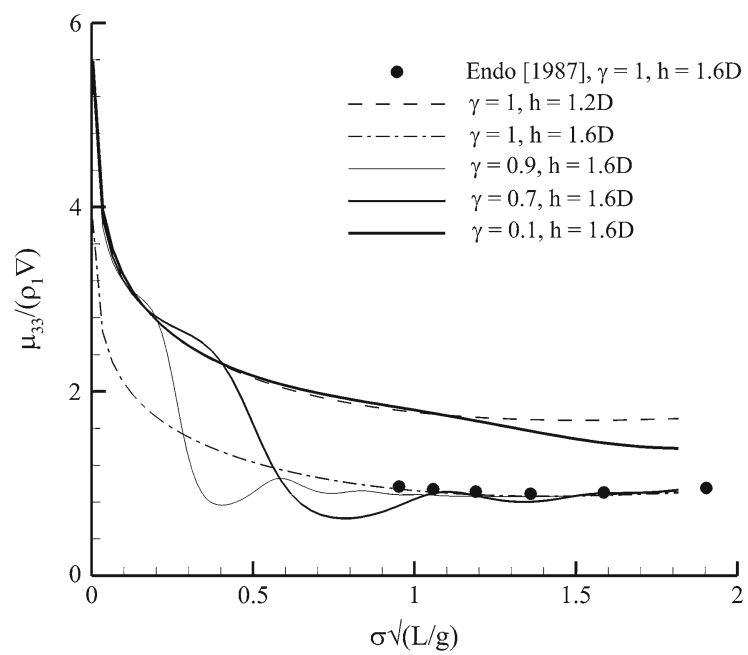

Fig. 6 Heave added mass of a rectangular box, $h_{1}=1.2 D$, $h_{2}=0.4 D$

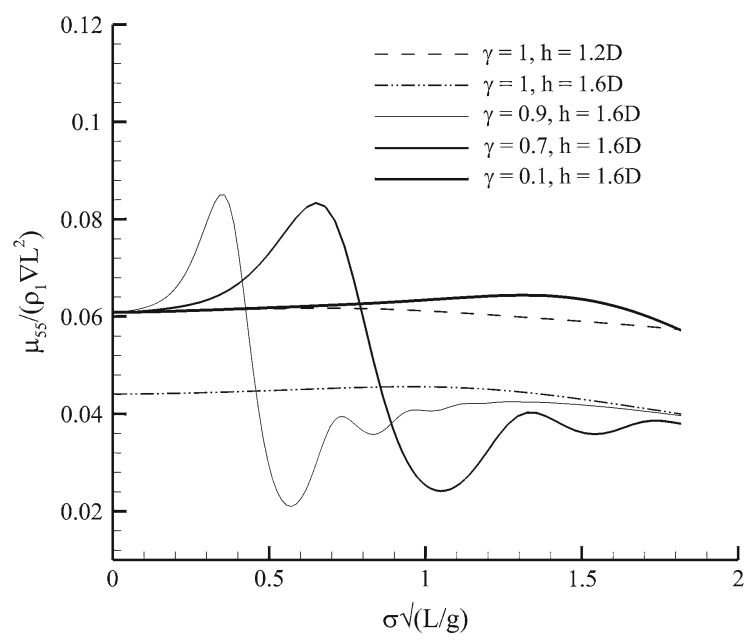

Fig. 8 Pitch added mass of a rectangular box, $h_{1}=1.2 D$, $h_{2}=0.4 D$

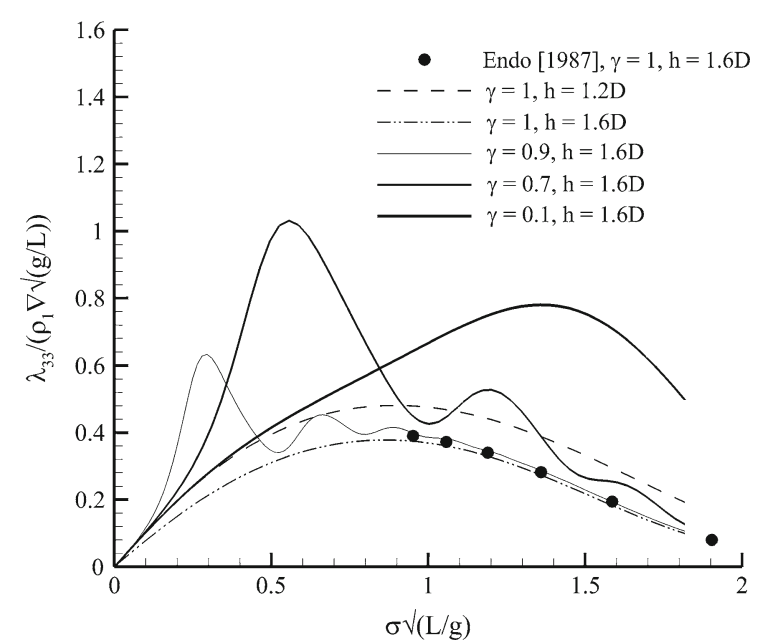

Fig. 7 Heave damping coefficient of a rectangular box, $h_{1}=1.2 D, h_{2}=0.4 D$

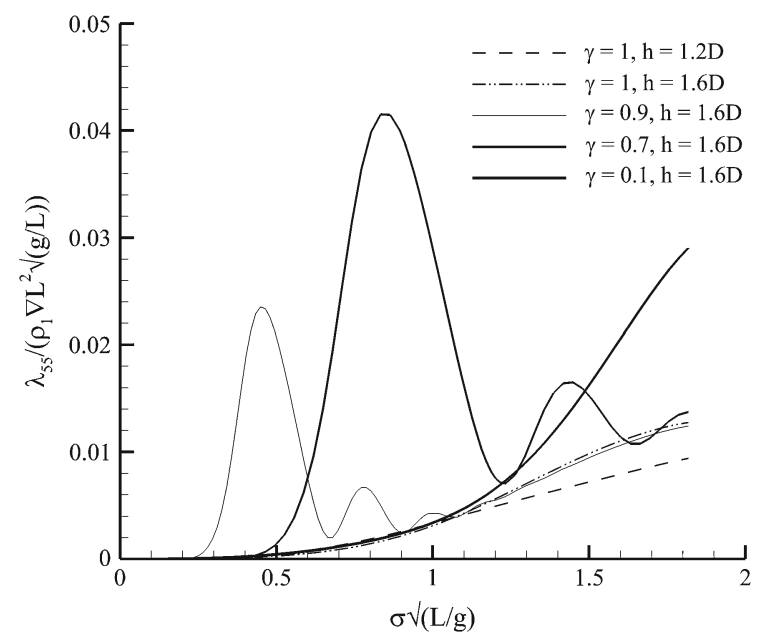

Fig. 9 Pitch damping coefficient of a rectangular box, $h_{1}=1.2 D, h_{2}=0.4 D$

lower frequencies, dramatic differences in the hydrodynamic coefficients can be seen, and they can be attributed to the presence of the internal waves. The frequency range in our calculations was chosen for ease of comparison with the results of Endo [28]. This range is smaller than the lowest "irregular frequencies" which are well known in the numerical solution of (113) using a source distribution (see [32]). Thus, the results shown here are smooth and do not contain the discontinuities characteristic of solution "breakdown" at the "irregular frequencies".

For any frequency of oscillation $\sigma$, the wavelength $\lambda_{1}$ of the surface-wave mode is larger than the wavelength $\lambda_{2}$ of the internal-wave mode. At high frequencies, $\lambda_{2}$ is small, and internal waves are less important. Also, the difference between $\lambda_{1}$ and $\lambda_{2}$ increases as $\gamma$ increases. This means that, as the density difference becomes very small, the effects of internal waves are concentrated in a small range of low frequencies. This can be seen in Fig. 7 where the heave damping coefficient for $\gamma=0.9$ exhibits a significant peak over a range of lower frequencies.

Figures 10, 11, 12, 13, 14 and 15 show the magnitude of the surge-, heave-, and pitch-exciting forces due to both the surface-wave mode and the internal-wave mode. These forces are normalized by the amplitude $a_{0}$ of the incident wave (see (119)). For the surface-wave mode, $a_{0}$ represents the amplitude of the incident surface wave, and simi- 


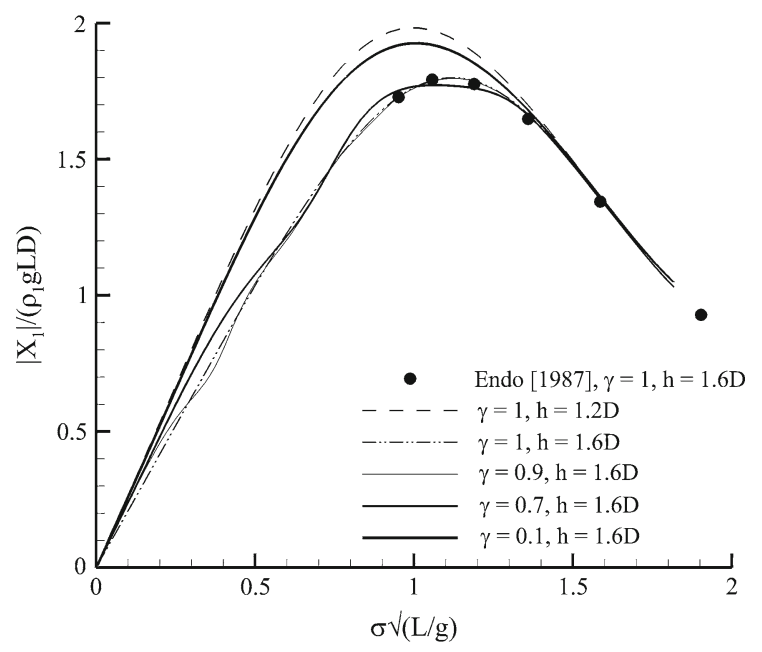

Fig. 10 Magnitude of surge exciting force, $n=1, h_{1}=1.2 D$, $h_{2}=0.4 D, \beta=0$

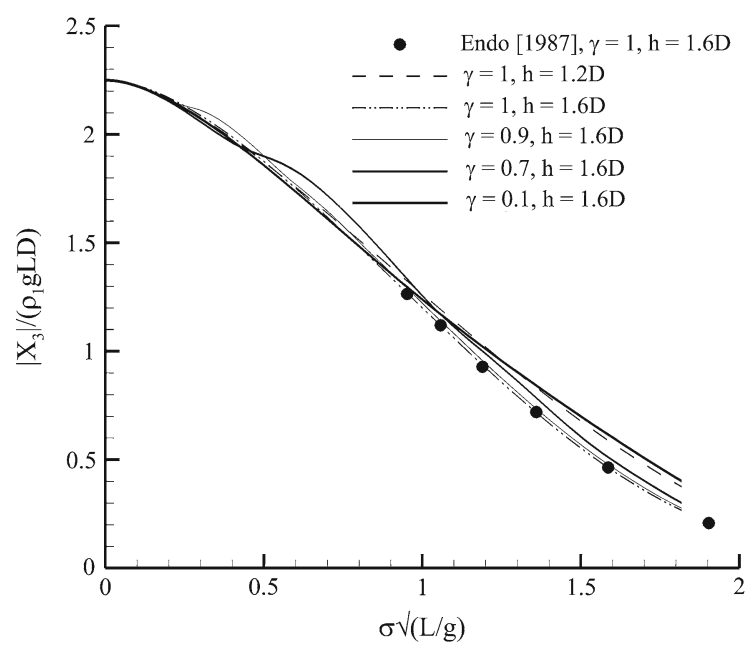

Fig. 12 Magnitude of heave exciting force, $n=1, h_{1}=1.2 D$, $h_{2}=0.4 D, \beta=0$

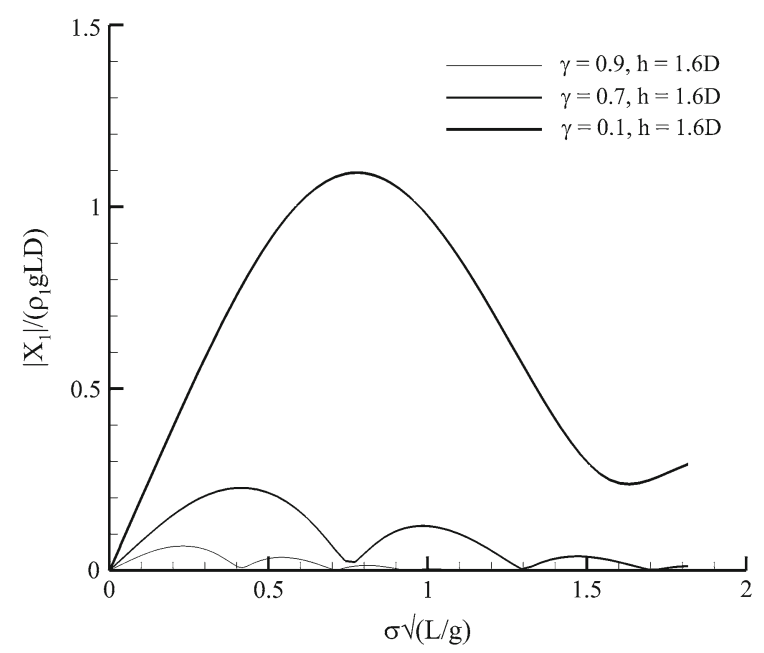

Fig. 11 Magnitude of surge exciting force, $n=2, h_{1}=1.2 D$, $h_{2}=0.4 D, \beta=0$

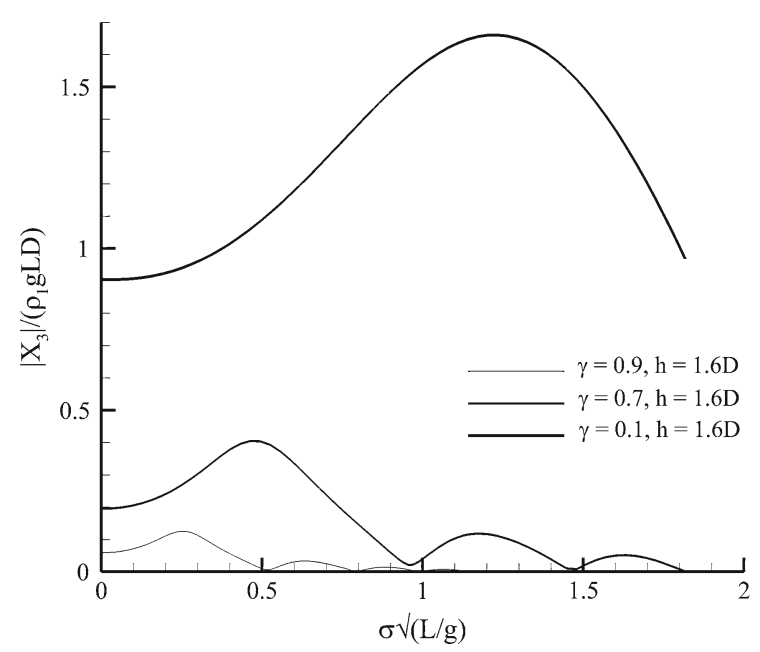

Fig. 13 Magnitude of heave exciting force, $n=2, h_{1}=1.2 D$, $h_{2}=0.4 D, \beta=0$

larly, for the internal-wave mode, $a_{0}$ is the amplitude of the incident internal wave. The surge- and heave-exciting forces for the surface-wave mode do not differ significantly from those due to surface waves in a single-layer fluid. The pitch-exciting moment, however, has larger oscillations especially for $\gamma=0.7$ and 0.9 . The exciting forces due to the internal-wave mode, which does not exist in a single-layer fluid, exhibit many peaks and troughs. These peaks and troughs and the oscillations seen earlier in the added-mass and damping coefficients are caused by the interaction between internal waves generated by the opposite edges of the rectangular box. The non-dimensional magnitude of the exciting forces increases as $\gamma$ decreases. This can be explained by the larger potential energy per unit amplitude of the incident internal waves when $\gamma$ is small.

Figures 16, 17, 18, 19, 20 and 21 show the motion amplitudes for both wave modes. Figure 18, in particular, shows the heave amplitude for the surface-wave mode and indicates that the presence of the lower fluid layer reduces 


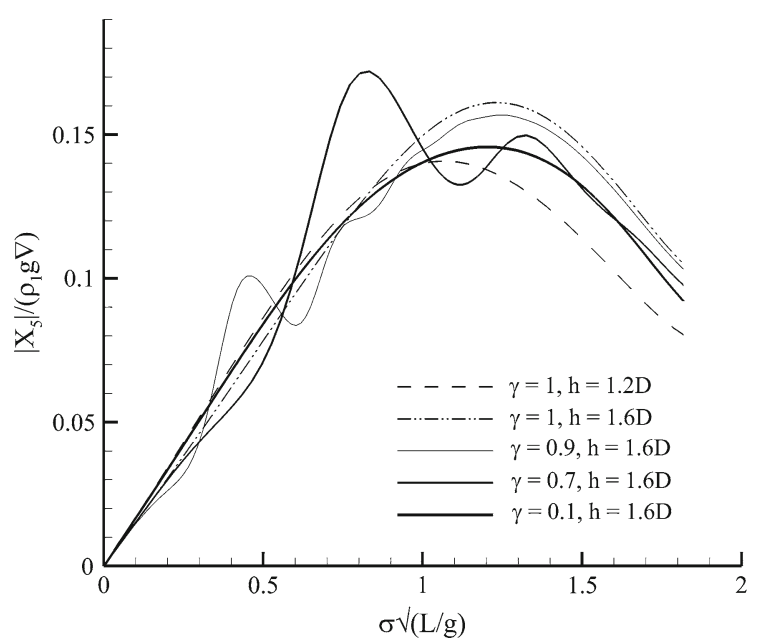

Fig. 14 Magnitude of pitch exciting moment, $n=1$, $h_{1}=1.2 D, h_{2}=0.4 D, \beta=0$

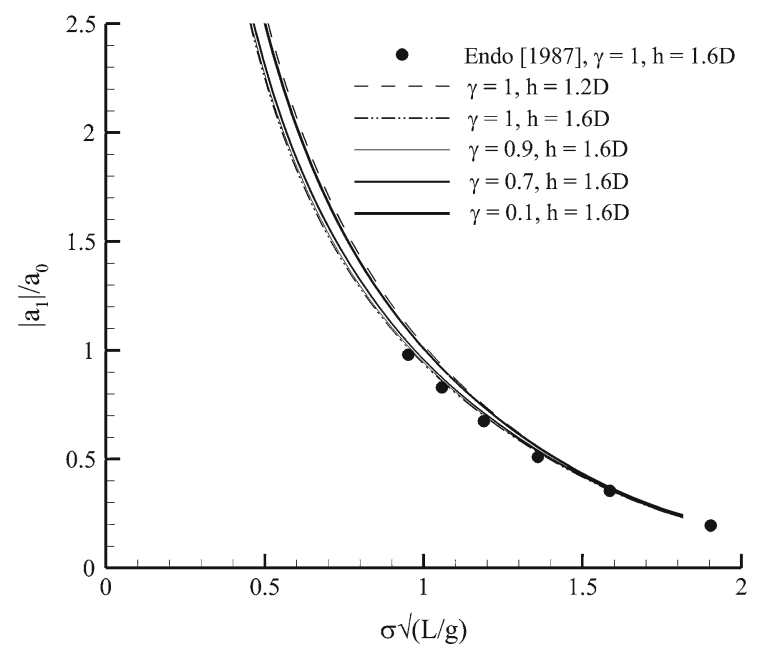

Fig. 16 Surge amplitude, surface-wave mode $(n=1)$, $h_{1}=1.2 D, h_{2}=0.4 D, \beta=0$

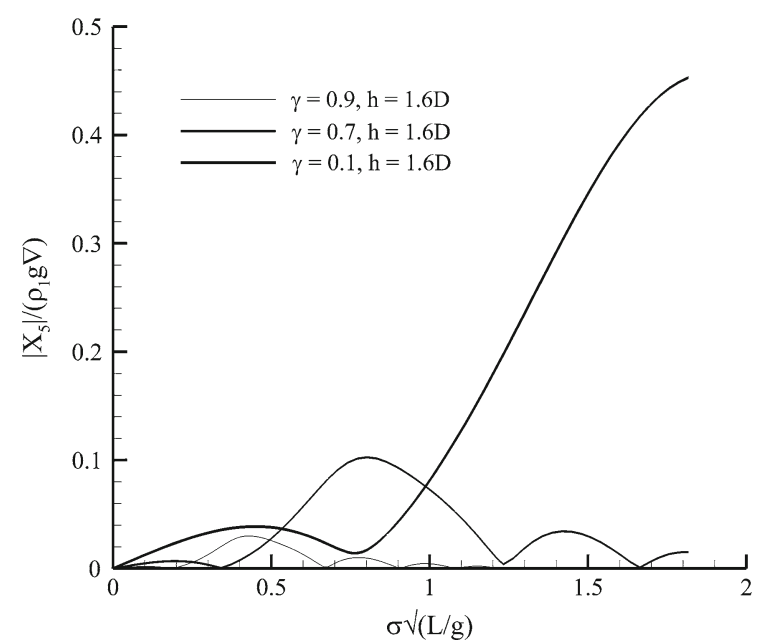

Fig. 15 Magnitude of pitch exciting moment, $n=2$, $h_{1}=1.2 D, h_{2}=0.4 D, \beta=0$

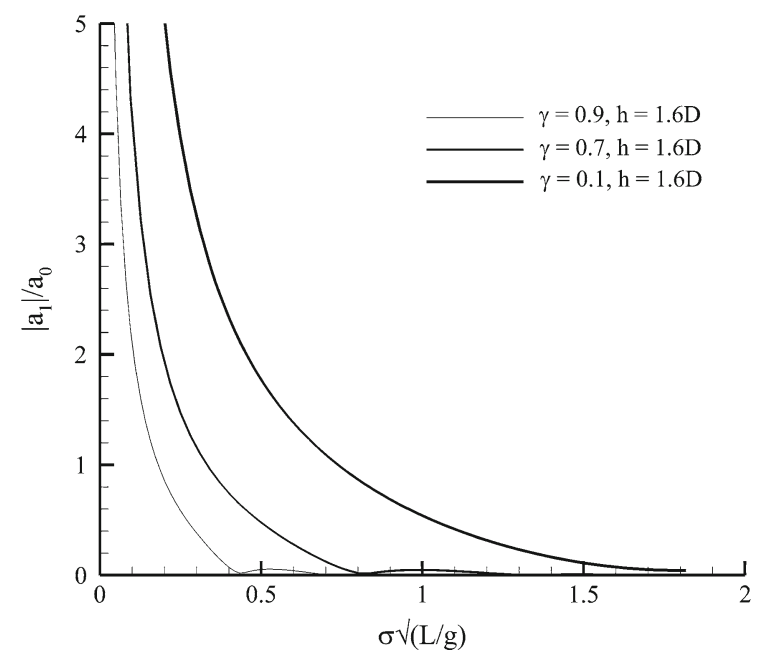

Fig. 17 Surge amplitude, internal-wave mode $(n=2)$, $h_{1}=1.2 D, h_{2}=0.4 D, \beta=0$

the heaving motion. Figure 21 shows the pitch amplitude for the internal-wave mode. Large pitching motion can be excited by the internal waves.

\section{Conclusions}

The velocity potentials of various unsteady sources have been derived in this paper for a two-layer fluid of finite depth. The two-layer fluid is bounded above by the free surface and below by a rigid, flat bottom. Although the sources treated here are restricted to the upper fluid layer, the same methodology can be extended in a straight-forward manner to obtain sources in the lower fluid. For marine vehicles, the draft is usually above the pycnocline or the mud layer, and only sources in the upper fluid are necessary. The potentials for a source of arbitrary strength, starting from rest and moving in an arbitrary path, are derived first by solving an initial, boundary-value 


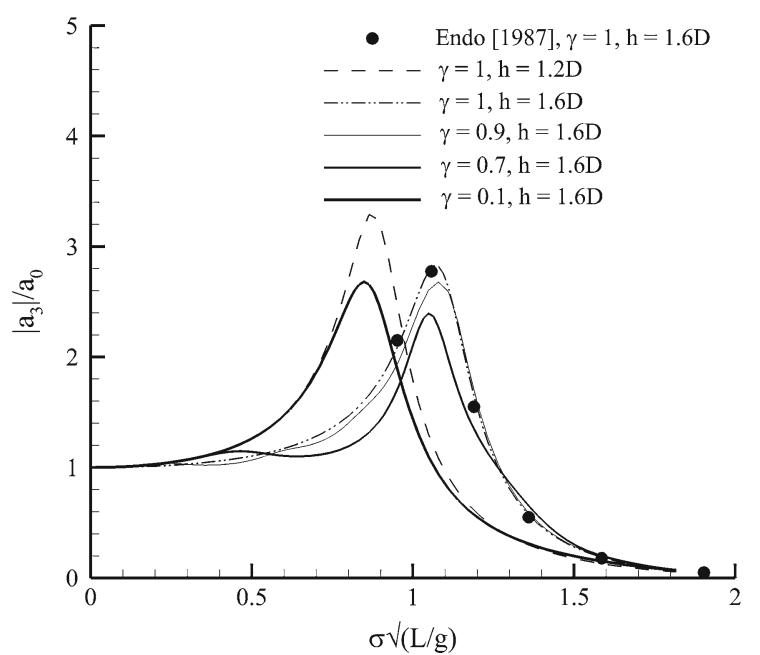

Fig. 18 Heave amplitude, surface-wave mode $(n=1)$, $h_{1}=1.2 D, h_{2}=0.4 D, \beta=0$

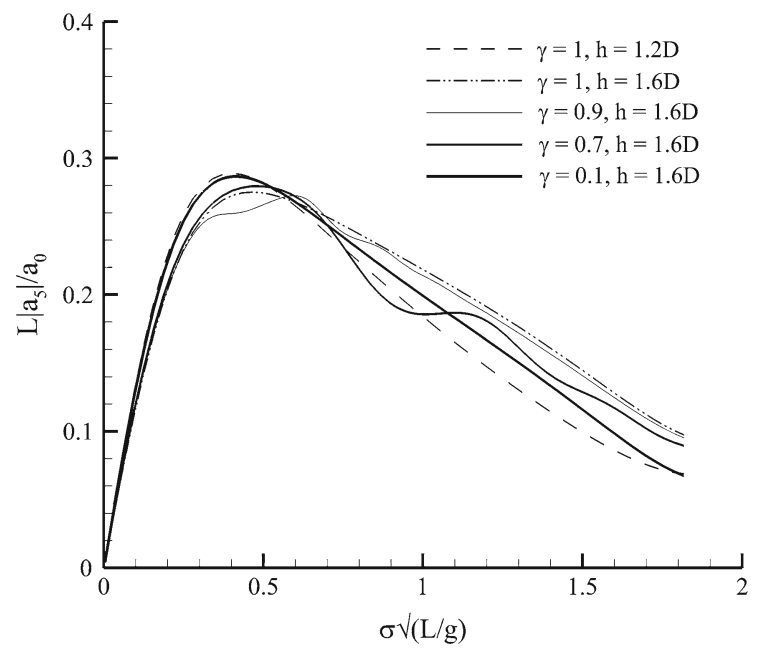

Fig. 20 Pitch amplitude, surface-wave mode $(n=1)$, $h_{1}=1.2 D, h_{2}=0.4 D, \beta=0$

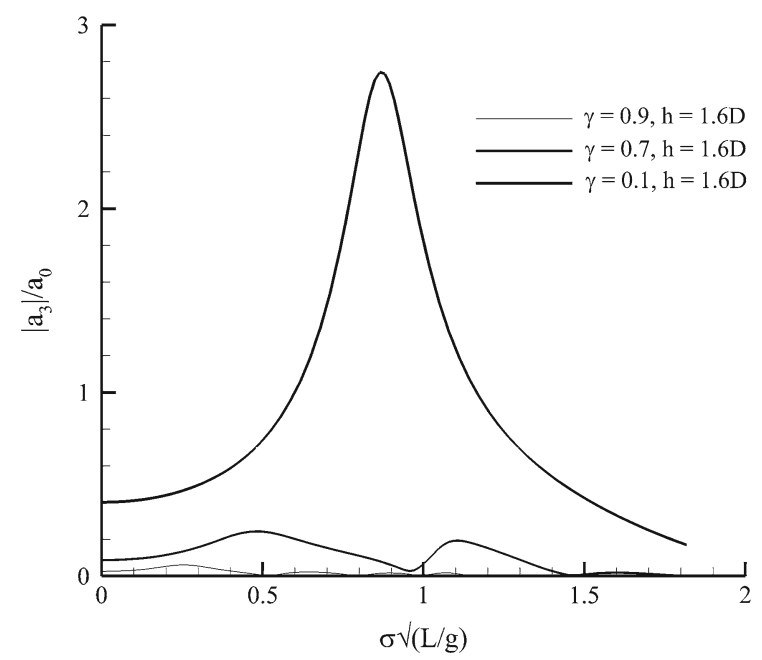

Fig. 19 Heave amplitude, internal-wave mode $(n=2)$, $h_{1}=1.2 D, h_{2}=0.4 D, \beta=0$

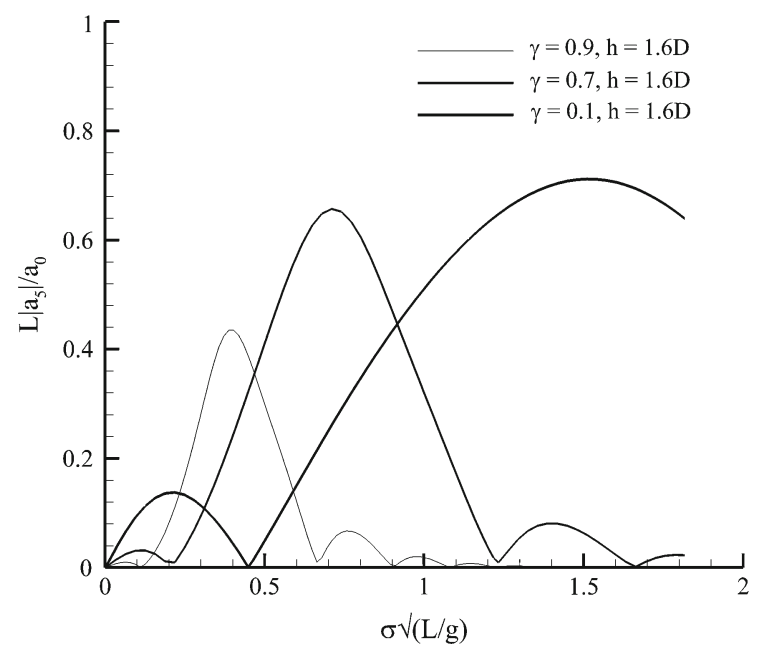

Fig. 21 Pitch amplitude, internal-wave mode $(n=2)$, $h_{1}=1.2 D, h_{2}=0.4 D, \beta=0$

problem using Laplace and Fourier transforms. The results are similar in form to the transient source in a single fluid. In fact, by equating the densities in the two layers, we can recover the potential of the single-fluid source. We can also obtain other sources of interest by starting with the transient source. It is trivial to derive sources with unit-step or delta-function strength so they have not been presented here. These sources are commonly used in time-domain calculations of ship motions. For frequency-domain calculations, oscillating sources with and without forward speed are used. They are derived from the transient source in Sects. 3 and 4 by specifying the appropriate strength and motion and performing the limit as time $t \rightarrow \infty$. These sources also have similar forms to their counterparts in a single fluid. Main differences are in the dependence on the vertical coordinate $z$, the dispersion relations, and the contribution from the internal waves.

The source potentials or Green functions given here can be implemented into existing panel codes to study the effects of internal waves on ship motions. As an example, the stationary, oscillating source in Sect. 3 is used in a 
low-order panel method to determine the motions of a floating barge under the action of surface and internal waves. For simplicity, the barge is modeled as a rectangular box, and results are obtained for density ratios $\gamma=\rho_{1} / \rho_{2}$ of $0.1,0.7,0.9,1$. This range covers both density stratification due to pycnoclines and mud layers. When $\gamma=1$, the two-layer fluid becomes a single fluid, and motion-response results are compared with those of Endo [28] for a single fluid. Excellent agreements are observed for added masses, damping coefficients, exciting forces, and motion amplitudes. For $\gamma$ other than unity, the motions of the barge can be affected by the presence of the fluid interface over a wide range of frequencies. For incident waves of the surface-wave mode, the heaving motion is reduced by the presence of the lower fluid layer. However, large pitching motion can be excited by incident waves of the internal-wave mode.

Acknowledgments The first author would like to acknowledge the support of the ILIR Program of the Naval Surface Warfare Center, Panama City Division. Partial support of this work, conducted under Office of Naval Research Grant No. N00014-09-1-1086 at The University of California at Berkeley is also gratefully acknowledged.

Open Access This article is distributed under the terms of the Creative Commons Attribution Noncommercial License which permits any noncommercial use, distribution, and reproduction in any medium, provided the original author(s) and source are credited.

\section{References}

1. Ekman VW (1904) On dead water. Norwegian North Polar Expedition, 1893-1896. Sci Results 5:1-150

2. Sretenskii LN (1959) On the wave resistance of ships in the presence of internal waves. Izv Akad Nauk C C C R Otdelenie Tekhnicheskikh 1:56-63

3. Hudimac AA (1961) Ship waves in a stratified ocean. J Fluid Mech 11:229-243

4. Michell JH (1898) Wave resistance of a ship. Phil Mag 5(45):106-123

5. Sabuncu T (1961) The theoretical wave resistance of a ship travelling under interfacial wave conditions. Norwegian Ship Model Experimental Tank, Trondheim, $124 \mathrm{pp}$

6. Uspenskii PN (1959) On the wave resistance of a ship in the presence of internal waves under conditions of finite depth. Akad Nauk USSR Tr Morskogo Gidrophizicheskogo Instituta 18:68-84

7. Miloh T, Tulin MP, Zilman G (1993) Dead-water effects of a ship moving in stratified seas. J Offshore Mech Arct Eng 115:105-110

8. Nguyen TC, Yeung RW (1997) Steady-wave systems in a two-layer fluid of finite depth. In: Proceedings of the 12th international workshop water waves and floating bodies, Marseilles, France, pp 115-119

9. Yeung RW, Nguyen TC (1999) Waves Generated by a moving source in a two-layer ocean of finite depth. J Eng Math 34(5):85-107

10. Sturova IV (1993) Scattering of surface and internal waves on submerged body. Comput Technol Novosibirsk 2:30-45

11. Sturova IV (1994) Plane problem of hydrodynamic rocking of a body submerged in a two-layer fluid without forward speed. Fluid Dyn 29:414-423

12. Sturova IV (1994) Hydrodynamic forces on a submerged cylinder advancing in waves of two-layer fluids. In: Proceedings of the 9th international workshop water waves and floating bodies, Kuju, Japan

13. Linton CM, McIver M (1995) The interaction of waves with horizontal cylinders in two-layer fluids. J Fluid Mech 304:213-229

14. Zilman G, Kagan L, Miloh T (1996) Hydrodynamics of a body moving over a mud layer-Part II: added-mass and damping coefficients. J Ship Res 40:39-45

15. Kashiwagi M (2005) Wave-induced motions of a body floating in a two-layer fluid. In: Proceedings of the 15 th international offshore and polar engineering conference, Seoul, Korea, vol 3, pp 358-365

16. Sturova IV, Syui Ch (2005) Hydrodynamic load associated with the oscillations of a cylinder at the interface in a two-layer fluid of finite depth. Fluid Dyn 40:273-281

17. Inglis RB, Price WG (1982) A three dimensional ship motion theory-comparison between theoretical predictions and experimental data of the hydrodynamic coefficients with forward speed. Trans R Inst Naval Archit 124:141-157

18. Wu GX, Eatock Taylor R (1989) The numerical solution of the motions of a ship advancing in waves. In: Proceedings of the 5th international conference on numerical ship hydrodynamics, Hiroshima, Japan

19. King BK, Beck RF, Magee AR (1988) Seakeeping calculations with forward speed using time-domain analysis. In: Proceedings of the 17th symposium on naval hydrodynamics, The Hague, Netherlands

20. Lin WM, Yue D (1991) Numerical solutions for large-amplitude ship motions in the time domain. In: Proceedings of the 18th symposium on naval hydrodynamics, Ann Arbor, MI

21. Zilman G, Miloh T (1994) Hydrodynamics of a body moving over a mud layer. In: Proceedings of the 20th symposium on naval hydrodynamics, Santa Barbara, CA

22. Gradshteyn IS, Ryzhik IM (1980) Table of integrals, series, and products. Academic Press, Orlando

23. Zilman G, Miloh T (1995) Hydrodynamics of a body moving over a mud layer-Part I: Wave resistance. J Ship Res 39:194-201

24. Wehausen JV, Laitone EV (1960) Surface waves. Handbuch der Physik 9:446-778 
25. Wehausen JV (1971) The motion of floating bodies. Annu Rev Fluid Mech 3:237-268

26. Newman JN (1992) The approximation of free-surface Green functions. In: Martin PA, Whickham GR (eds) Wave asymptotics. Cambridge University Press, Cambridge

27. Conte SD, Boor C (1980) Elementary numerical analysis. McGraw-Hill, New York

28. Endo H (1987) Shallow-water effect on the motions of three-dimensional bodies in waves. J Ship Res 31:34-40

29. Yeung RW (1982) Numerical methods in free surface flows. Annu Rev Fluid Mech 14:395-442

30. Hess JL, Smith AMO (1964) Calculation of nonlifting potential flow about arbitrary three-dimensional bodies. J Ship Res 8:22-44

31. Hamilton JA, Yeung RW (1997) Shell-function solutions for three-dimensional nonlinear body motion problems. Schiffstechnik 44:62-70

32. John F (1950) On the motion of floating bodies-II. Simple harmonic motions. Commun Pure Appl Math 3:45-101 